\title{
Separated boundary value problems for second-order impulsive $q$-integro-difference equations
}

Chatthai Thaiprayoon ${ }^{1}$, Jessada Tariboon ${ }^{1 *}$ and Sotiris K Ntouyas ${ }^{2}$

"Correspondence:

jessadat@kmutnb.ac.th

'Department of Mathematics,

Faculty of Applied Science, King

Mongkut's University of Technology,

North Bangkok, Bangkok, Thailand

Full list of author information is

available at the end of the article

\begin{abstract}
This article studies the existence and uniqueness of solutions for a boundary value problem of nonlinear second-order impulsive $q_{k}$-integro-difference equations with separated boundary conditions. Several new results are obtained by applying a variety of fixed point theorems. Some examples are presented to illustrate the results.
\end{abstract} MSC: 26A33; 39A13; 34A37

Keywords: $q_{k}$-derivative; $q_{k}$-integral; impulsive $q_{k}$-difference equation; existence; uniqueness; fixed point theorems

\section{Introduction}

In this paper, we study the separated boundary value problem for impulsive $q_{k}$-integrodifference equation of the following form:

$$
\left\{\begin{array}{l}
D_{q_{k}}^{2} x(t)=f\left(t, x(t),\left(S_{q_{k}} x\right)(t)\right), \quad t \in J:=[0, T], t \neq t_{k}, \\
\Delta x\left(t_{k}\right)=I_{k}\left(x\left(t_{k}\right)\right), \quad k=1,2, \ldots, m, \\
D_{q_{k}} x\left(t_{k}^{+}\right)-D_{q_{k-1}} x\left(t_{k}\right)=I_{k}^{*}\left(x\left(t_{k}\right)\right), \quad k=1,2, \ldots, m, \\
x(0)+D_{q_{0}} x(0)=0, \quad x(T)+D_{q_{m}} x(T)=0,
\end{array}\right.
$$

where $0=t_{0}<t_{1}<t_{2}<\cdots<t_{k}<\cdots<t_{m}<t_{m+1}=T, f: J \times \mathbb{R}^{2} \rightarrow \mathbb{R}$,

$$
\left(S_{q_{k}} x\right)(t)=\int_{t_{k}}^{t} \phi(t, s) x(s) d_{q_{k}} s, \quad t \in\left(t_{k}, t_{k+1}\right], k=0,1,2, \ldots, m,
$$

$\phi: J \times J \rightarrow[0, \infty)$ is a continuous function, $I_{k}, I_{k}^{*} \in C(\mathbb{R}, \mathbb{R}), \Delta x\left(t_{k}\right)=x\left(t_{k}^{+}\right)-x\left(t_{k}\right)$ for $k=$ $1,2, \ldots, m, x\left(t_{k}^{+}\right)=\lim _{h \rightarrow 0} x\left(t_{k}+h\right)$ and $0<q_{k}<1$ for $k=0,1,2, \ldots, m$.

The notions of $q_{k}$-derivative and $q_{k}$-integral on finite intervals were introduced in [1]. For a fixed $k \in \mathbb{N} \cup\{0\}$ let $J_{k}:=\left[t_{k}, t_{k+1}\right] \subset \mathbb{R}$ be an interval and $0<q_{k}<1$ be a constant. We define $q_{k}$-derivative of a function $f: J_{k} \rightarrow \mathbb{R}$ at a point $t \in J_{k}$ as follows.

Definition 1.1 Assume $f: J_{k} \rightarrow \mathbb{R}$ is a continuous function and let $t \in J_{k}$. Then the expression

$$
D_{q_{k}} f(t)=\frac{f(t)-f\left(q_{k} t+\left(1-q_{k}\right) t_{k}\right)}{\left(1-q_{k}\right)\left(t-t_{k}\right)}, \quad t \neq t_{k}, \quad D_{q_{k}} f\left(t_{k}\right)=\lim _{t \rightarrow t_{k}} D_{q_{k}} f(t)
$$

is called the $q_{k}$-derivative of function $f$ at $t$.

\section{囪 Springer}

O2014 Thaiprayoon et al.; licensee Springer. This is an Open Access article distributed under the terms of the Creative Commons Attribution License (http://creativecommons.org/licenses/by/2.0), which permits unrestricted use, distribution, and reproduction in any medium, provided the original work is properly cited. 
We say that $f$ is $q_{k}$-differentiable on $J_{k}$ provided $D_{q_{k}} f(t)$ exists for all $t \in J_{k}$. Note that if $t_{k}=0$ and $q_{k}=q$ in (1.3), then $D_{q_{k}} f=D_{q} f$, where $D_{q}$ is the well-known $q$-derivative of the function $f(t)$ defined by

$$
D_{q} f(t)=\frac{f(t)-f(q t)}{(1-q) t} .
$$

In addition, we should define the higher $q_{k}$-derivative of functions.

Definition 1.2 Let $f: J_{k} \rightarrow \mathbb{R}$ be a continuous function, we call the second-order $q_{k}$ derivative $D_{q_{k}}^{2} f$ provided $D_{q_{k}} f$ is $q_{k}$-differentiable on $J_{k}$ with $D_{q_{k}}^{2} f=D_{q_{k}}\left(D_{q_{k}} f\right): J_{k} \rightarrow \mathbb{R}$. Similarly, we define higher order $q_{k}$-derivative $D_{q_{k}}^{n}: J_{k} \rightarrow \mathbb{R}$.

The $q_{k}$-integral is defined as follows.

Definition 1.3 Assume $f: J_{k} \rightarrow \mathbb{R}$ is a continuous function. Then the $q_{k}$-integral is defined by

$$
\int_{t_{k}}^{t} f(s) d_{q_{k}} s=\left(1-q_{k}\right)\left(t-t_{k}\right) \sum_{n=0}^{\infty} q_{k}^{n} f\left(q_{k}^{n} t+\left(1-q_{k}^{n}\right) t_{k}\right)
$$

for $t \in J_{k}$. Moreover, if $a \in\left(t_{k}, t\right)$ then the definite $q_{k}$-integral is defined by

$$
\begin{aligned}
\int_{a}^{t} f(s) d_{q_{k}} s= & \int_{t_{k}}^{t} f(s) d_{q_{k}} s-\int_{t_{k}}^{a} f(s) d_{q_{k}} s \\
= & \left(1-q_{k}\right)\left(t-t_{k}\right) \sum_{n=0}^{\infty} q_{k}^{n} f\left(q_{k}^{n} t+\left(1-q_{k}^{n}\right) t_{k}\right) \\
& -\left(1-q_{k}\right)\left(a-t_{k}\right) \sum_{n=0}^{\infty} q_{k}^{n} f\left(q_{k}^{n} a+\left(1-q_{k}^{n}\right) t_{k}\right) .
\end{aligned}
$$

Note that if $t_{k}=0$ and $q_{k}=q$, then (1.5) reduces to $q$-integral of a function $f(t)$, defined by $\int_{0}^{t} f(s) d_{q} s=(1-q) t \sum_{n=0}^{\infty} q^{n} f\left(q^{n} t\right)$ for $t \in[0, \infty)$.

For the basic properties of $q_{k}$-derivative and $q_{k}$-integral we refer to [1].

The book by Kac and Cheung [2] covers many of the fundamental aspects of the quantum calculus. In recent years, the topic of $q$-calculus has attracted the attention of several researchers and a variety of new results can be found in the papers [3-15] and the references cited therein.

Impulsive differential equations serve as basic models to study the dynamics of processes that are subject to sudden changes in their states. Recent development in this field has been motivated by many applied problems, such as control theory, population dynamics and medicine. For some recent works on the theory of impulsive differential equations, we refer the interested reader to the monographs [16-18].

In this paper we prove an existence and uniqueness result for the impulsive boundary value problem (1.1) by using Banach's contraction mapping principle and three existence results by applying Schaefer's, Krasnoselskii's fixed point theorems and the LeraySchauder Nonlinear Alternative. The rest of this paper is organized as follows: In Section 2 we present an auxiliary lemma which is used to convert the impulsive boundary 
value problem (1.1) into an equivalent integral equation. The main results are given in Section 3, while examples illustrating the results are presented in Section 4.

\section{An auxiliary lemma}

Let $J=[0, T], J_{0}=\left[t_{0}, t_{1}\right], J_{k}=\left(t_{k}, t_{k+1}\right]$ for $k=1,2, \ldots, m$. Let $P C(J, \mathbb{R})=\{x: J \rightarrow \mathbb{R}: x(t)$ is continuous everywhere except for some $t_{k}$ at which $x\left(t_{k}^{+}\right)$and $x\left(t_{k}^{-}\right)$exist and $x\left(t_{k}^{-}\right)=x\left(t_{k}\right)$, $k=1,2, \ldots, m\} . P C(J, \mathbb{R})$ is a Banach space with the norm $\|x\|_{P C}=\sup \{|x(t)| ; t \in J\}$.

We now consider the following linear case:

$$
\left\{\begin{array}{l}
D_{q_{k}}^{2} x(t)=h(t), \quad t \in J, t \neq t_{k}, \\
\Delta x\left(t_{k}\right)=I_{k}\left(x\left(t_{k}\right)\right), \quad k=1,2, \ldots, m, \\
D_{q_{k}} x\left(t_{k}^{+}\right)-D_{q_{k-1}} x\left(t_{k}\right)=I_{k}^{*}\left(x\left(t_{k}\right)\right), \quad k=1,2, \ldots, m, \\
x(0)+D_{q_{0}} x(0)=0, \quad x(T)+D_{q_{m}} x(T)=0,
\end{array}\right.
$$

where $h: J \rightarrow \mathbb{R}$ is a continuous function.

Lemma 2.1 The unique solution of problem (2.1) is given by

$$
\begin{aligned}
x(t)= & \left(\frac{1-t}{T}\right) \sum_{k=1}^{m}\left(\int_{t_{k-1}}^{t_{k}} \int_{t_{k-1}}^{s} h(r) d_{q_{k-1}} r d_{q_{k-1}} s+I_{k}\left(x\left(t_{k}\right)\right)\right) \\
& +\left(\frac{1-t}{T}\right) \sum_{k=1}^{m}\left(\int_{t_{k-1}}^{t_{k}} h(s) d_{q_{k-1}} s+I_{k}^{*}\left(x\left(t_{k}\right)\right)\right)\left(T-t_{k}+1\right) \\
& +\left(\frac{1-t}{T}\right) \int_{t_{m}}^{T} \int_{t_{m}}^{s} h(r) d_{q_{m}} r d_{q_{m}} s+\left(\frac{1-t}{T}\right) \int_{t_{m}}^{T} h(s) d_{q_{m}} s \\
& +\sum_{0<t_{k}<t}\left(\int_{t_{k-1}}^{t_{k}} \int_{t_{k-1}}^{s} h(r) d_{q_{k-1}} r d_{q_{k-1}} s+I_{k}\left(x\left(t_{k}\right)\right)\right) \\
& +\sum_{0<t_{k}<t}\left(\int_{t_{k-1}}^{t_{k}} h(s) d_{q_{k-1}} s+I_{k}^{*}\left(x\left(t_{k}\right)\right)\right)\left(t-t_{k}\right)+\int_{t_{k}}^{t} \int_{t_{k}}^{s} h(r) d_{q_{k}} r d_{q_{k}} s,
\end{aligned}
$$

with $\sum_{i=a}^{b}(\cdot)=0$ for $a>b$.

Proof Taking the $q_{0}$-integral for the first equation of (2.1), for $t \in J_{0}$, we have

$$
D_{q_{0}} x(t)=D_{q_{0}} x(0)+\int_{0}^{t} h(s) d_{q_{0}} s,
$$

which leads to

$$
D_{q_{0}} x\left(t_{1}\right)=D_{q_{0}} x(0)+\int_{0}^{t_{1}} h(s) d_{q_{0}} s .
$$

For $t \in J_{0}$ we get by $q_{0}$-integrating (2.3),

$$
\begin{aligned}
x(t) & =x(0)+D_{q_{0}} x(0) t+\int_{0}^{t} \int_{0}^{s} h(r) d_{q_{0}} r d_{q_{0}} s \\
& :=A+B t+\int_{0}^{t} \int_{0}^{s} h(r) d_{q_{0}} r d_{q_{0}} s, \quad \text { if } A=x(0), B=D_{q_{0}} x(0) .
\end{aligned}
$$


In particular, for $t=t_{1}$, we obtain

$$
x\left(t_{1}\right)=A+B t_{1}+\int_{0}^{t_{1}} \int_{0}^{s} h(r) d_{q_{0}} r d_{q_{0}} s
$$

For $t \in J_{1}=\left(t_{1}, t_{2}\right], q_{1}$-integrating (2.1), we have

$$
D_{q_{1}} x(t)=D_{q_{1}} x\left(t_{1}^{+}\right)+\int_{t_{1}}^{t} h(s) d_{q_{1}} s
$$

Using the third condition of (2.1) with (2.4), it follows that

$$
D_{q_{1}} x(t)=B+\int_{0}^{t_{1}} h(s) d_{q_{0}} s+I_{1}^{*}\left(x\left(t_{1}\right)\right)+\int_{t_{1}}^{t} h(s) d_{q_{1}} s .
$$

Taking $q_{1}$-integral to (2.6) for $t \in J_{1}$, we obtain

$$
\begin{aligned}
x(t)= & x\left(t_{1}^{+}\right)+\left[B+\int_{0}^{t_{1}} h(s) d_{q_{0}} s+I_{1}^{*}\left(x\left(t_{1}\right)\right)\right]\left(t-t_{1}\right) \\
& +\int_{t_{1}}^{t} \int_{t_{1}}^{s} h(r) d_{q_{1}} r d_{q_{1}} s .
\end{aligned}
$$

Applying the second equation of (2.1) with (2.5) and (2.7), we get

$$
\begin{aligned}
x(t)= & A+B t_{1}+\int_{0}^{t_{1}} \int_{0}^{s} h(r) d_{q_{0}} r d_{q_{0}} s+I_{1}\left(x\left(t_{1}\right)\right) \\
& +\left[B+\int_{0}^{t_{1}} h(s) d_{q_{0}} s+I_{1}^{*}\left(x\left(t_{1}\right)\right)\right]\left(t-t_{1}\right)+\int_{t_{1}}^{t} \int_{t_{1}}^{s} h(r) d_{q_{1}} r d_{q_{1}} s \\
= & A+B t+\int_{0}^{t_{1}} \int_{0}^{s} h(r) d_{q_{0}} r d_{q_{0}} s+I_{1}\left(x\left(t_{1}\right)\right) \\
& +\left[\int_{0}^{t_{1}} h(s) d_{q_{0}} s+I_{1}^{*}\left(x\left(t_{1}\right)\right)\right]\left(t-t_{1}\right)+\int_{t_{1}}^{t} \int_{t_{1}}^{s} h(r) d_{q_{1}} r d_{q_{1}} s .
\end{aligned}
$$

Repeating the above process, for $t \in J_{k}$, we get

$$
\begin{aligned}
x(t)= & A+B t \\
& +\sum_{0<t_{k}<t}\left(\int_{t_{k-1}}^{t_{k}} \int_{t_{k-1}}^{s} h(r) d_{q_{k-1}} r d_{q_{k-1}} s+I_{k}\left(x\left(t_{k}\right)\right)\right) \\
& +\sum_{0<t_{k}<t}\left(\int_{t_{k-1}}^{t_{k}} h(s) d_{q_{k-1}} s+I_{k}^{*}\left(x\left(t_{k}\right)\right)\right)\left(t-t_{k}\right) \\
& +\int_{t_{k}}^{t} \int_{t_{k}}^{s} h(r) d_{q_{k}} r d_{q_{k}} s .
\end{aligned}
$$

From the first boundary condition of (2.1) (i.e. $\left.x(0)+D_{q_{0}} x(0)=0\right)$ and (2.8), we have

$$
A+B=0 .
$$


Also, the second boundary condition of (2.1) (i.e. $\left.x(T)+D_{q_{m}} x(T)=0\right)$ and (2.8), yields

$$
\begin{aligned}
A+ & B(T+1)+\sum_{k=1}^{m}\left(\int_{t_{k-1}}^{t_{k}} \int_{t_{k-1}}^{s} h(r) d_{q_{k-1}} r d_{q_{k-1}} s+I_{k}\left(x\left(t_{k}\right)\right)\right) \\
& +\sum_{k=1}^{m}\left(\int_{t_{k-1}}^{t_{k}} h(s) d_{q_{k-1}} s+I_{k}^{*}\left(x\left(t_{k}\right)\right)\right)\left(T-t_{k}+1\right) \\
& +\int_{t_{m}}^{T} \int_{t_{m}}^{s} h(r) d_{q_{m}} r d_{q_{m}} s+\int_{t_{m}}^{T} h(s) d_{q_{m}} s=0 .
\end{aligned}
$$

From (2.9) and (2.10), we have that

$$
\begin{aligned}
B= & -\frac{1}{T} \sum_{k=1}^{m}\left(\int_{t_{k-1}}^{t_{k}} \int_{t_{k-1}}^{s} h(r) d_{q_{k-1}} r d_{q_{k-1}} s+I_{k}\left(x\left(t_{k}\right)\right)\right) \\
& -\frac{1}{T} \sum_{i=1}^{m}\left(\int_{t_{k-1}}^{t_{k}} h(s) d_{q_{k-1}} s+I_{k}^{*}\left(x\left(t_{k}\right)\right)\right)\left(T-t_{k}+1\right) \\
& -\frac{1}{T} \int_{t_{m}}^{T} \int_{t_{m}}^{s} h(r) d_{q_{m}} r d_{q_{m}} s-\frac{1}{T} \int_{t_{m}}^{T} h(s) d_{q_{m}} s,
\end{aligned}
$$

which implies

$$
\begin{aligned}
A= & \frac{1}{T} \sum_{k=1}^{m}\left(\int_{t_{k-1}}^{t_{k}} \int_{t_{k-1}}^{s} h(r) d_{q_{k-1}} r d_{q_{k-1}} s+I_{k}\left(x\left(t_{k}\right)\right)\right) \\
& +\frac{1}{T} \sum_{k=1}^{m}\left(\int_{t_{k-1}}^{t_{k}} h(s) d_{q_{k-1}} s+I_{k}^{*}\left(x\left(t_{k}\right)\right)\right)\left(T-t_{k}+1\right) \\
& +\frac{1}{T} \int_{t_{m}}^{T} \int_{t_{m}}^{s} h(r) d_{q_{m}} r d_{q_{m}} s+\frac{1}{T} \int_{t_{m}}^{T} h(s) d_{q_{m}} s .
\end{aligned}
$$

Substituting constants $A$ and $B$ into (2.8), we obtain (2.2) as requested.

\section{Main results}

In view of Lemma 2.1, we define an operator $\mathcal{A}: P C(J, \mathbb{R}) \rightarrow P C(J, \mathbb{R})$ by

$$
\begin{aligned}
(\mathcal{A} x)(t)= & \left(\frac{1-t}{T}\right) \sum_{k=1}^{m}\left(\int_{t_{k-1}}^{t_{k}} \int_{t_{k-1}}^{s} f\left(r, x(r),\left(S_{q_{k-1}} x\right)(r)\right) d_{q_{k-1}} r d_{q_{k-1}} s+I_{k}\left(x\left(t_{k}\right)\right)\right) \\
& +\left(\frac{1-t}{T}\right) \sum_{k=1}^{m}\left(\int_{t_{k-1}}^{t_{k}} f\left(s, x(s),\left(S_{q_{k-1}} x\right)(s)\right) d_{q_{k-1}} s+I_{k}^{*}\left(x\left(t_{k}\right)\right)\right)\left(T-t_{k}+1\right) \\
& +\left(\frac{1-t}{T}\right) \int_{t_{m}}^{T} \int_{t_{m}}^{s} f\left(r, x(r),\left(S_{q_{m}} x\right)(r)\right) d_{q_{m}} r d_{q_{m}} s \\
& +\left(\frac{1-t}{T}\right) \int_{t_{m}}^{T} f\left(s, x(s),\left(S_{q_{m}} x\right)(s)\right) d_{q_{m}} s \\
& +\sum_{0<t_{k}<t}\left(\int_{t_{k-1}}^{t_{k}} \int_{t_{k-1}}^{s} f\left(r, x(r),\left(S_{q_{k-1}} x\right)(r)\right) d_{q_{k-1}} r d_{q_{k-1}} s+I_{k}\left(x\left(t_{k}\right)\right)\right)
\end{aligned}
$$




$$
\begin{aligned}
& +\sum_{0<t_{k}<t}\left(\int_{t_{k-1}}^{t_{k}} f\left(s, x(s),\left(S_{q_{k-1}} x\right)(s)\right) d_{q_{k-1}} s+I_{k}^{*}\left(x\left(t_{k}\right)\right)\right)\left(t-t_{k}\right) \\
& +\int_{t_{k}}^{t} \int_{t_{k}}^{s} f\left(r, x(r),\left(S_{q_{k}} x\right)(r)\right) d_{q_{k}} r d_{q_{k}} s .
\end{aligned}
$$

It should be noticed that problem (1.1) has solutions if and only if the operator $\mathcal{A}$ has fixed points.

Our first result is an existence and uniqueness result for the impulsive boundary value problem (1.1) by using the Banach contraction mapping principle.

Let $\phi_{0}=\max \{\phi(t, s):(t, s) \in J \times J\}$. Further, for convenience we set

$$
\begin{aligned}
\omega= & \frac{1+2 T}{T} \sum_{k=1}^{m+1}\left[\frac{L_{1}\left(t_{k}-t_{k-1}\right)^{2}}{1+q_{k-1}}+\frac{\phi_{0} L_{2}\left(t_{k}-t_{k-1}\right)^{3}}{1+q_{k-1}+q_{k-1}^{2}}\right] \\
& +\frac{1+T}{T} \sum_{k=1}^{m+1}\left[L_{1}\left(t_{k}-t_{k-1}\right)+\frac{\phi_{0} L_{2}\left(t_{k}-t_{k-1}\right)^{2}}{1+q_{k-1}}\right] \\
& +\frac{1+2 T}{T} \sum_{k=1}^{m}\left(T-t_{k}\right)\left[L_{1}\left(t_{k}-t_{k-1}\right)+\frac{\phi_{0} L_{2}\left(t_{k}-t_{k-1}\right)^{2}}{1+q_{k-1}}\right] \\
& +\frac{m L_{3}(1+2 T)}{T}+\frac{m L_{4}(1+T)}{T}+\frac{L_{4}(1+2 T)}{T} \sum_{k=1}^{m}\left(T-t_{k}\right),
\end{aligned}
$$

and

$$
\begin{aligned}
\lambda_{0}= & \frac{M_{1}(1+2 T)}{T} \sum_{k=1}^{m+1} \frac{\left(t_{k}-t_{k-1}\right)^{2}}{1+q_{k-1}}+\frac{M_{1}(1+T)}{T} \sum_{k=1}^{m+1}\left(t_{k}-t_{k-1}\right) \\
& +\frac{M_{1}(1+2 T)}{T} \sum_{k=1}^{m}\left(T-t_{k}\right)\left(t_{k}-t_{k-1}\right)+\frac{m M_{2}(1+2 T)}{T} \\
& +\frac{m M_{3}(1+T)}{T}+\frac{M_{3}(1+2 T)}{T} \sum_{k=1}^{m}\left(T-t_{k}\right) .
\end{aligned}
$$

Theorem 3.1 Assume that:

$\left(\mathrm{H}_{1}\right)$ The function $f: J \times \mathbb{R}^{2} \rightarrow \mathbb{R}$ is continuous and there exist constants $L_{1}, L_{2}>0$ such that

$$
\left|f\left(t, x,\left(S_{q_{k}} x\right)\right)-f\left(t, y,\left(S_{q_{k}} y\right)\right)\right| \leq L_{1}|x-y|+L_{2}\left(S_{q_{k}}|x-y|\right),
$$

for each $t \in J$ and $x, y \in \mathbb{R}, k=0,1,2, \ldots, m$.

$\left(\mathrm{H}_{2}\right)$ The functions $I_{k}, I_{k}^{*}: \mathbb{R} \rightarrow \mathbb{R}$ are continuous and there exist constants $L_{3}, L_{4}>0$ such that

$$
\left|I_{k}(x)-I_{k}(y)\right| \leq L_{3}|x-y| \quad \text { and } \quad\left|I_{k}^{*}(x)-I_{k}^{*}(y)\right| \leq L_{4}|x-y|
$$

for each $x, y \in \mathbb{R}, k=1,2, \ldots, m$. 
If

$\omega \leq \delta<1$

where $\omega$ is defined by (3.2), and $\delta>0$, then the boundary value problem (1.1) has a unique solution on $J$.

Proof We transform the boundary value problem (1.1) into a fixed point problem, $x=\mathcal{A} x$, where the operator $\mathcal{A}$ is defined by (3.1). By using the Banach contraction mapping principle, we shall show that $\mathcal{A}$ has a fixed point which is the unique solution of the boundary value problem (1.1).

Let $M_{1}, M_{2}$, and $M_{3}$ be nonnegative constants such that $\sup _{t \in J}|f(t, 0,0)|=M_{1}$, $\sup \left\{\left|I_{k}(0)\right|: k=1,2, \ldots, m\right\}=M_{2}$, and $\sup \left\{\left|I_{k}^{*}(0)\right|: k=1,2, \ldots, m\right\}=M_{3}$. By choosing a constant $R$ as

$$
R \geq \frac{\lambda_{0}}{1-\varepsilon}
$$

where $\delta \leq \varepsilon<1$ and $\lambda_{0}$ defined by (3.3), we will show that $\mathcal{A} B_{R} \subset B_{R}$, where a ball $B_{R}$ is defined by $B_{R}=\{x \in P C(J, \mathbb{R}):\|x\| \leq R\}$. For $x \in B_{R}$, we have

$$
\begin{aligned}
\|\mathcal{A} x\| & \sup _{t \in J}\left\{\left(\frac{1-t}{T}\right) \sum_{k=1}^{m}\left(\int_{t_{k-1}}^{t_{k}} \int_{t_{k-1}}^{s} f\left(r, x(r),\left(S_{q_{k-1}} x\right)(r)\right) d_{q_{k-1}} r d_{q_{k-1}} s+I_{k}\left(x\left(t_{k}\right)\right)\right)\right. \\
& +\left(\frac{1-t}{T}\right) \sum_{k=1}^{m}\left(\int_{t_{k-1}}^{t_{k}} f\left(s, x(s),\left(S_{q_{k-1}} x\right)(s)\right) d_{q_{k-1}} s+I_{k}^{*}\left(x\left(t_{k}\right)\right)\right)\left(T-t_{k}+1\right) \\
& +\left(\frac{1-t}{T}\right) \int_{t_{m}}^{T} \int_{t_{m}}^{s} f\left(r, x(r),\left(S_{q_{m}} x\right)(r)\right) d_{q_{m}} r d_{q_{m}} s \\
& +\left(\frac{1-t}{T}\right) \int_{t_{m}}^{T} f\left(s, x(s),\left(S_{q_{m}} x\right)(s)\right) d_{q_{m}} s \\
& +\sum_{0<t_{k}<t}\left(\int_{t_{k-1}}^{t_{k}} \int_{t_{k-1}}^{s} f\left(r, x(r),\left(S_{q_{k-1}} x\right)(r)\right) d_{q_{k-1}} r d_{q_{k-1}} s+I_{k}\left(x\left(t_{k}\right)\right)\right) \\
& +\sum_{0<t_{k}<t}\left(\int_{t_{k-1}}^{t_{k}} f\left(s, x(s),\left(S_{q_{k-1}} x\right)(s)\right) d_{q_{k-1}} s+I_{k}^{*}\left(x\left(t_{k}\right)\right)\right)\left(t-t_{k}\right) \\
& \left.+\int_{t_{k}}^{t} \int_{t_{k}}^{s} f\left(r, x(r),\left(S_{q_{k}} x\right)(r)\right) d_{q_{k}} r d_{q_{k}} s\right\} \\
\leq & \left(\frac{1+T}{T}\right) \sum_{k=1}^{m}\left(\int_{t_{k-1}}^{t_{k}} \int_{t_{k-1}}^{s}\left|f\left(r, x(r),\left(S_{q_{k-1}} x\right)(r)\right)\right| d_{q_{k-1}} r d_{q_{k-1}} s+\left|I_{k}\left(x\left(t_{k}\right)\right)\right|\right) \\
+ & \left(\frac{1+T}{T}\right) \sum_{k=1}^{m}\left(\int_{t_{k-1}}^{t_{k}}\left|f\left(s, x(s),\left(S_{q_{k-1}} x\right)(s)\right)\right| d_{q_{k-1}} s+\left|I_{k}^{*}\left(x\left(t_{k}\right)\right)\right|\right)\left(T-t_{k}+1\right) \\
+ & \left(\frac{1+T}{T}\right) \int_{t_{m}}^{T} \int_{t_{m}}^{s}\left|f\left(r, x(r),\left(S_{q_{m}} x\right)(r)\right)\right| d_{q_{m}} r d_{q_{m}} s \\
&
\end{aligned}
$$


Thaiprayoon et al. Advances in Difference Equations 2014, 2014:88

Page 8 of 23

http://www.advancesindifferenceequations.com/content/2014/1/88

$$
\begin{aligned}
& +\left(\frac{1+T}{T}\right) \int_{t_{m}}^{T}\left|f\left(s, x(s),\left(S_{q_{m}} x\right)(s)\right)\right| d_{q_{m}} s \\
& +\sum_{k=1}^{m}\left(\int_{t_{k-1}}^{t_{k}} \int_{t_{k-1}}^{s}\left|f\left(r, x(r),\left(S_{q_{k-1}} x\right)(r)\right)\right| d_{q_{k-1}} r d_{q_{k-1}} s+\left|I_{k}\left(x\left(t_{k}\right)\right)\right|\right) \\
& +\sum_{k=1}^{m}\left(\int_{t_{k-1}}^{t_{k}}\left|f\left(s, x(s),\left(S_{q_{k-1}} x\right)(s)\right)\right| d_{q_{k-1}} s+\left|I_{k}^{*}\left(x\left(t_{k}\right)\right)\right|\right)\left(T-t_{k}\right) \\
& +\int_{t_{m}}^{T} \int_{t_{m}}^{s}\left|f\left(r, x(r),\left(S_{q_{m}} x\right)(r)\right)\right| d_{q_{m}} r d_{q_{m}} s \\
& \leq \frac{1+2 T}{T} \sum_{k=1}^{m+1} \int_{t_{k-1}}^{t_{k}} \int_{t_{k-1}}^{s}\left(\left|f\left(r, x(r),\left(S_{q_{k-1}} x\right)(r)\right)-f(r, 0,0)\right|+|f(r, 0,0)|\right) d_{q_{k-1}} r d_{q_{k-1}} s \\
& +\frac{1+T}{T} \sum_{k=1}^{m+1} \int_{t_{k-1}}^{t_{k}}\left(\left|f\left(s, x(s),\left(S_{q_{k-1}} x\right)(s)\right)-f(s, 0,0)\right|+|f(s, 0,0)|\right) d_{q_{k-1}} s \\
& +\frac{1+2 T}{T} \sum_{k=1}^{m}\left(T-t_{k}\right) \int_{t_{k-1}}^{t_{k}}\left(\left|f\left(s, x(s),\left(S_{q_{k-1}} x\right)(s)\right)-f(s, 0,0)\right|+|f(s, 0,0)|\right) d_{q_{k-1}} s \\
& +\frac{1+2 T}{T} \sum_{k=1}^{m}\left(\left|I_{k}\left(x\left(t_{k}\right)\right)-I_{k}(0)\right|+\left|I_{k}(0)\right|\right) \\
& +\frac{1+T}{T} \sum_{k=1}^{m}\left(\left|I_{k}^{*}\left(x\left(t_{k}\right)\right)-I_{k}^{*}(0)\right|+\left|I_{k}^{*}(0)\right|\right) \\
& +\frac{1+2 T}{T} \sum_{k=1}^{m}\left(\left|I_{k}^{*}\left(x\left(t_{k}\right)\right)-I_{k}^{*}(0)\right|+\left|I_{k}^{*}(0)\right|\right)\left(T-t_{k}\right) \\
& \leq \frac{1+2 T}{T} \sum_{k=1}^{m+1} \int_{t_{k-1}}^{t_{k}} \int_{t_{k-1}}^{s}\left(L_{1} R+\phi_{0} L_{2} R \int_{t_{k-1}}^{r} d_{q_{k-1}} u+M_{1}\right) d_{q_{k-1}} r d_{q_{k-1}} s \\
& +\frac{1+T}{T} \sum_{k=1}^{m+1} \int_{t_{k-1}}^{t_{k}}\left(L_{1} R+\phi_{0} L_{2} R \int_{t_{k-1}}^{s} d_{q_{k-1}} r+M_{1}\right) d_{q_{k-1}} s \\
& +\frac{1+2 T}{T} \sum_{k=1}^{m}\left(T-t_{k}\right) \int_{t_{k-1}}^{t_{k}}\left(L_{1} R+\phi_{0} L_{2} R \int_{t_{k-1}}^{s} d_{q_{k-1}} r+M_{1}\right) d_{q_{k-1}} s \\
& +\frac{1+2 T}{T} \sum_{k=1}^{m}\left(L_{3} R+M_{2}\right)+\frac{1+T}{T} \sum_{k=1}^{m}\left(L_{4} R+M_{3}\right) \\
& +\frac{1+2 T}{T} \sum_{k=1}^{m}\left(L_{4} R+M_{3}\right)\left(T-t_{k}\right) \\
& \leq \frac{1+2 T}{T} \sum_{k=1}^{m+1}\left[\frac{L_{1} R\left(t_{k}-t_{k-1}\right)^{2}}{1+q_{k-1}}+\frac{\phi_{0} L_{2} R\left(t_{k}-t_{k-1}\right)^{3}}{1+q_{k-1}+q_{k-1}^{2}}+\frac{M_{1}\left(t_{k}-t_{k-1}\right)^{2}}{1+q_{k-1}}\right] \\
& +\frac{1+T}{T} \sum_{k=1}^{m+1}\left[L_{1} R\left(t_{k}-t_{k-1}\right)+\frac{\phi_{0} L_{2} R\left(t_{k}-t_{k-1}\right)^{2}}{1+q_{k-1}}+M_{1}\left(t_{k}-t_{k-1}\right)\right] \\
& +\frac{1+2 T}{T} \sum_{k=1}^{m}\left(T-t_{k}\right)\left[L_{1} R\left(t_{k}-t_{k-1}\right)+\frac{\phi_{0} L_{2} R\left(t_{k}-t_{k-1}\right)^{2}}{1+q_{k-1}}+M_{1}\left(t_{k}-t_{k-1}\right)\right]
\end{aligned}
$$




$$
\begin{aligned}
& +\frac{1+2 T}{T} \sum_{k=1}^{m}\left(L_{3} R+M_{2}\right)+\frac{1+T}{T} \sum_{k=1}^{m}\left(L_{4} R+M_{3}\right)+\frac{1+2 T}{T} \sum_{k=1}^{m}\left(L_{4} R+M_{3}\right)\left(T-t_{k}\right) \\
= & \omega R+\lambda_{0} \leq(\delta+1-\varepsilon) R \leq R,
\end{aligned}
$$

which implies that $\mathcal{A} B_{R} \subset B_{R}$.

For any $x, y \in P C(J, \mathbb{R})$ and for each $t \in J$, we have

$$
\begin{aligned}
\mid \mathcal{A} x(t) & -\mathcal{A} y(t) \mid \\
\leq & \frac{1+2 T}{T} \sum_{k=1}^{m+1} \int_{t_{k-1}}^{t_{k}} \int_{t_{k-1}}^{s}\left(\left|f\left(r, x(r),\left(S_{q_{k-1}} x\right)(r)\right)-f\left(r, y(r),\left(S_{q_{k-1}} y\right)(r)\right)\right|\right) d_{q_{k-1}} r d_{q_{k-1}} s \\
& +\frac{1+T}{T} \sum_{k=1}^{m+1} \int_{t_{k-1}}^{t_{k}}\left(\left|f\left(s, x(s),\left(S_{q_{k-1}} x\right)(s)\right)-f\left(s, y(s),\left(S_{q_{k-1}} y\right)(s)\right)\right|\right) d_{q_{k-1}} s \\
& +\frac{1+2 T}{T} \sum_{k=1}^{m}\left(T-t_{k}\right) \int_{t_{k-1}}^{t_{k}}\left(\left|f\left(s, x(s),\left(S_{q_{k-1}} x\right)(s)\right)-f\left(s, x(s),\left(S_{q_{k-1}} x\right)(s)\right)\right|\right) d_{q_{k-1}} s \\
& +\frac{1+2 T}{T} \sum_{k=1}^{m}\left(\left|I_{k}\left(x\left(t_{k}\right)\right)-I_{k}\left(y\left(t_{k}\right)\right)\right|\right)+\frac{1+T}{T} \sum_{k=1}^{m}\left(\left|I_{k}^{*}\left(x\left(t_{k}\right)\right)-I_{k}^{*}\left(y\left(t_{k}\right)\right)\right|\right) \\
& +\frac{1+2 T}{T} \sum_{k=1}^{m}\left(\left|I_{k}^{*}\left(x\left(t_{k}\right)\right)-I_{k}^{*}\left(y\left(t_{k}\right)\right)\right|\right)\left(T-t_{k}\right) \\
\leq & \frac{1+2 T}{T} \sum_{k=1}^{m+1}\left[\frac{L_{1}\left(t_{k}-t_{k-1}\right)^{2}}{1+q_{k-1}}+\frac{\phi_{0} L_{2}\left(t_{k}-t_{k-1}\right)^{3}}{1+q_{k-1}+q_{k-1}^{2}}\right]\|x-y\| \\
& +\frac{1+T}{T} \sum_{k=1}^{m+1}\left[L_{1}\left(t_{k}-t_{k-1}\right)+\frac{\phi_{0} L_{2}\left(t_{k}-t_{k-1}\right)^{2}}{1+q_{k-1}}\right]\|x-y\| \\
& +\frac{1+2 T}{T} \sum_{k=1}^{m}\left(T-t_{k}\right)\left[L_{1}\left(t_{k}-t_{k-1}\right)+\frac{\phi_{0} L_{2}\left(t_{k}-t_{k-1}\right)^{2}}{1+q_{k-1}}\right]\|x-y\| \\
& +\frac{m(1+2 T) L_{3}}{T}\|x-y\|+\frac{m(1+T) L_{4}}{T}\|x-y\|+\frac{(1+2 T) L_{4}\|x-y\|}{T} \sum_{k=1}^{m}\left(T-t_{k}\right) \\
= & \|x-y\|,
\end{aligned}
$$

which implies that $\|\mathcal{A} x-\mathcal{A} y\| \leq \omega\|x-y\|$. As $\omega<1, \mathcal{A}$ is a contraction. Therefore, by the Banach contraction mapping principle, we find that $\mathcal{A}$ has a fixed point which is the unique solution of problem (1.1). This completes the proof.

The second existence result is based on Schaefer's fixed point theorem.

\section{Theorem 3.2 Assume that:}

$\left(\mathrm{H}_{3}\right) f: J \times \mathbb{R}^{2} \rightarrow \mathbb{R}$ is a continuous function and there exists a constant $N_{1}>0$ such that

$$
\left|f\left(t, x,\left(S_{q_{k}} x\right)\right)\right| \leq N_{1}
$$

for each $t \in J$ and all $x \in \mathbb{R}, k=0,1,2, \ldots, m$. 
$\left(\mathrm{H}_{4}\right)$ The functions $I_{k}, I_{k}^{*}: \mathbb{R} \rightarrow \mathbb{R}$ are continuous and there exist constants $N_{2}, N_{3}>0$ such that

$$
\left|I_{k}(x)\right| \leq N_{2} \text { and }\left|I_{k}^{*}(x)\right| \leq N_{3}
$$

for all $x \in \mathbb{R}, k=1,2, \ldots, m$.

Then the boundary value problem (1.1) has at least one solution on J.

Proof We will use Schaefer's fixed point theorem to prove that $\mathcal{A}$, defined by (3.1), has a fixed point. We divide the proof into four steps.

Step 1: Continuity of $\mathcal{A}$.

Let $\left\{x_{n}\right\}$ be a sequence such that $x_{n} \rightarrow x$ in $P C(J, \mathbb{R})$. Since $f$ is a continuous function on $J \times \mathbb{R}^{2}$ and $I_{k}, I_{k}^{*}$ are continuous functions on $\mathbb{R}$ for $k=1,2, \ldots$, we have

$$
f\left(t, x_{n}(t),\left(S_{q_{k}} x_{n}\right)(t)\right) \rightarrow f\left(t, x(t),\left(S_{q_{k}} x\right)(t)\right),
$$

and $I_{k}\left(x_{n}\left(t_{k}\right)\right) \rightarrow I_{k}\left(x\left(t_{k}\right)\right), I_{k}^{*}\left(x_{n}\left(t_{k}\right)\right) \rightarrow I_{k}^{*}\left(x\left(t_{k}\right)\right)$ for $k=1,2, \ldots$, as $n \rightarrow \infty$.

Then, for each $t \in J$, we get

$$
\begin{aligned}
\left|\left(\mathcal{A} x_{n}\right)(t)-(\mathcal{A} x)(t)\right| & \left(\frac{1-t}{T}\right) \sum_{k=1}^{m}\left(\int_{t_{k-1}}^{t_{k}} \int_{t_{k-1}}^{s} \mid f\left(r, x_{n}(r),\left(S_{q_{k-1}} x_{n}\right)(r)\right)\right. \\
& \left.-f\left(r, x(r),\left(S_{q_{k-1}} x\right)(r)\right)\left|d_{q_{k-1}} r d_{q_{k-1}} s+\right| I_{k}\left(x_{n}\left(t_{k}\right)\right)-I_{k}\left(x\left(t_{k}\right)\right) \mid\right) \\
& +\left(\frac{1-t}{T}\right) \sum_{k=1}^{m}\left(\int_{t_{k-1}}^{t_{k}}\left|f\left(s, x_{n}(s),\left(S_{q_{k-1}} x_{n}\right)(s)\right)-f\left(s, x(s),\left(S_{q_{k-1}} x\right)(s)\right)\right| d_{q_{k-1}} s\right. \\
& \left.+\left|I_{k}^{*}\left(x_{n}\left(t_{k}\right)\right)-I_{k}^{*}\left(x\left(t_{k}\right)\right)\right|\right)\left(T-t_{k}+1\right) \\
& +\left(\frac{1-t}{T}\right) \int_{t_{m}}^{T} \int_{t_{m}}^{s}\left|f\left(r, x_{n}(r),\left(S_{q_{m}} x_{n}\right)(r)\right)-f\left(r, x(r),\left(S_{q_{m}} x\right)(r)\right)\right| d_{q_{m}} r d_{q_{m}} s \\
& +\left(\frac{1-t}{T}\right) \int_{t_{m}}^{T}\left|f\left(s, x_{n}(s),\left(S_{q_{m}} x_{n}\right)(s)\right)-f\left(s, x(s),\left(S_{q_{m}} x\right)(s)\right)\right| d_{q_{m}} s \\
& +\sum_{0<t_{k}<t}\left(\int_{t_{k-1}}^{t_{k}} \int_{t_{k-1}}^{s}\left|f\left(r, x_{n}(r),\left(S_{q_{k-1}} x_{n}\right)(r)\right)-f\left(r, x(r),\left(S_{q_{k-1}} x\right)(r)\right)\right| d_{q_{k-1}} r d_{q_{k-1}} s\right. \\
& \left.+\left|I_{k}\left(x_{n}\left(t_{k}\right)\right)-I_{k}\left(x\left(t_{k}\right)\right)\right|\right) \\
& +\sum_{0<t_{k}<t}\left(\int_{t_{k-1}}^{t_{k}}\left|f\left(s, x_{n}(s),\left(S_{q_{k-1}} x_{n}\right)(s)\right)-f\left(s, x(s),\left(S_{q_{k-1}} x\right)(s)\right)\right| d_{q_{k-1}} s\right. \\
+ & \left.\left|I_{k}^{*}\left(x_{n}\left(t_{k}\right)\right)-I_{k}^{*}\left(x\left(t_{k}\right)\right)\right|\right)\left(t-t_{t_{k}}\right) \\
+ & f\left(r, x_{n}(r),\left(S_{q_{k}} x_{n}\right)(r)\right)-f\left(r, x(r),\left(S_{q_{k}} x\right)(r)\right) \mid d_{q_{k}} r d_{q_{k}} s, \\
&
\end{aligned}
$$

which gives $\left\|\mathcal{A} x_{n}-\mathcal{A} x\right\| \rightarrow 0$ as $n \rightarrow \infty$. This means that $\mathcal{A}$ is continuous. 
Step 2: $\mathcal{A}$ maps bounded sets into bounded sets in $P C(J, \mathbb{R})$.

So, let us prove that for any $r>0$, there exists a positive constant $\rho$ such that for each $x \in B_{r}=\{x \in P C(J, \mathbb{R}):\|x\| \leq r\}$, we have $\|\mathcal{A} x\| \leq \rho$. For any $x \in B_{r}$, we have

$$
\begin{aligned}
& |(\mathcal{A} x)(t)| \leq \frac{|1-t|}{T} \sum_{k=1}^{m}\left(\int_{t_{k-1}}^{t_{k}} \int_{t_{k-1}}^{s}\left|f\left(r, x(r),\left(S_{q_{k-1}} x\right)(r)\right)\right| d_{q_{k-1}} r d_{q_{k-1}} s+\left|I_{k}\left(x\left(t_{k}\right)\right)\right|\right) \\
& +\frac{|1-t|}{T} \sum_{k=1}^{m}\left(\int_{t_{k-1}}^{t_{k}}\left|f\left(s, x(s),\left(S_{q_{k-1}} x\right)(s)\right)\right| d_{q_{k-1}} s+\left|I_{k}^{*}\left(x\left(t_{k}\right)\right)\right|\right)\left(T-t_{k}+1\right) \\
& +\frac{|1-t|}{T} \int_{t_{m}}^{T} \int_{t_{m}}^{s}\left|f\left(r, x(r),\left(S_{q_{m}} x\right)(r)\right)\right| d_{q_{m}} r d_{q_{m}} s \\
& +\frac{|1-t|}{T} \int_{t_{m}}^{T}\left|f\left(s, x(s),\left(S_{q_{m}} x\right)(s)\right)\right| d_{q_{m}} s \\
& +\sum_{k=1}^{m}\left(\int_{t_{k-1}}^{t_{k}} \int_{t_{k-1}}^{s}\left|f\left(r, x(r),\left(S_{q_{k-1}} x\right)(r)\right)\right| d_{q_{k-1}} r d_{q_{k-1}} s+\left|I_{k}\left(x\left(t_{k}\right)\right)\right|\right) \\
& +\sum_{k=1}^{m}\left(\int_{t_{k-1}}^{t_{k}}\left|f\left(s, x(s),\left(S_{q_{k-1}} x\right)(s)\right)\right| d_{q_{k-1}} s+\left|I_{k}^{*}\left(x\left(t_{k}\right)\right)\right|\right)\left(T-t_{k}\right) \\
& +\int_{t_{m}}^{T} \int_{t_{m}}^{s}\left|f\left(r, x(r),\left(S_{q_{m}} x\right)(r)\right)\right| d_{q_{m}} r d_{q_{m}} s \\
& \leq \frac{1+2 T}{T} \sum_{k=1}^{m+1} \int_{t_{k-1}}^{t_{k}} \int_{t_{k-1}}^{s} N_{1} d_{q_{k-1}} r d_{q_{k-1}} s+\frac{1+T}{T} \sum_{k=1}^{m+1} \int_{t_{k-1}}^{t_{k}} N_{1} d_{q_{k-1}} s \\
& +\frac{1+2 T}{T} \sum_{k=1}^{m}\left(T-t_{k}\right) \int_{t_{k-1}}^{t_{k}} N_{1} d_{q_{k-1}} s+\frac{1+2 T}{T} \sum_{k=1}^{m} N_{2} \\
& +\frac{1+T}{T} \sum_{k=1}^{m} N_{3}+\frac{1+2 T}{T} \sum_{k=1}^{m} N_{3}\left(T-t_{k}\right) \\
& \leq \frac{1+2 T}{T} \sum_{k=1}^{m+1}\left[\frac{N_{1}\left(t_{k}-t_{k-1}\right)^{2}}{1+q_{k-1}}\right]+\frac{1+T}{T} \sum_{k=1}^{m+1}\left[N_{1}\left(t_{k}-t_{k-1}\right)\right] \\
& +\frac{1+2 T}{T} \sum_{k=1}^{m}\left(T-t_{k}\right)\left[N_{1}\left(t_{k}-t_{k-1}\right)\right]+\frac{m(1+2 T) N_{2}}{T} \\
& +\frac{m(1+T) N_{3}}{T}+\frac{(1+2 T) N_{3}}{T} \sum_{k=1}^{m}\left(T-t_{k}\right) \\
& :=\rho \text {. }
\end{aligned}
$$

Hence, we deduce that $\|\mathcal{A} x\| \leq \rho$.

Step 3: $\mathcal{A}$ maps bounded sets into equicontinuous sets of $P C(J, \mathbb{R})$.

Let $\tau_{1}, \tau_{2} \in J_{i}$ for some $i \in\{0,1,2, \ldots, m\}, \tau_{1}<\tau_{2}, B_{r}$ be a bounded set of $P C(J, \mathbb{R})$ as in Step 2, and let $x \in B_{r}$. Then we have

$$
\begin{aligned}
& \left|(\mathcal{A} x)\left(\tau_{2}\right)-(\mathcal{A} x)\left(\tau_{1}\right)\right| \\
& \quad \leq \frac{\left|\tau_{2}-\tau_{1}\right|}{T} \sum_{k=1}^{m}\left(\int_{t_{k-1}}^{t_{k}} \int_{t_{k-1}}^{s}\left|f\left(r, x(r),\left(S_{q_{k-1}} x\right)(r)\right)\right| d_{q_{k-1}} r d_{q_{k-1}} s+\left|I_{k}\left(x\left(t_{k}\right)\right)\right|\right)
\end{aligned}
$$




$$
\begin{aligned}
& +\frac{\left|\tau_{2}-\tau_{1}\right|}{T} \sum_{k=1}^{m}\left(\int_{t_{k-1}}^{t_{k}}\left|f\left(s, x(s),\left(S_{q_{k-1}} x\right)(s)\right)\right| d_{q_{k-1}} s+\left|I_{k}^{*}\left(x\left(t_{k}\right)\right)\right|\right)\left(T-t_{k}+1\right) \\
& +\frac{\left|\tau_{2}-\tau_{1}\right|}{T} \int_{t_{m}}^{T} \int_{t_{m}}^{s}\left|f\left(r, x(r),\left(S_{q_{m}} x\right)(r)\right)\right| d_{q_{m}} r d_{q_{m}} s \\
& +\frac{\left|\tau_{2}-\tau_{1}\right|}{T} \int_{t_{m}}^{T}\left|f\left(s, x(s),\left(S_{q_{m}} x\right)(s)\right)\right| d_{q_{m}} s \\
& +\left|\tau_{2}-\tau_{1}\right| \sum_{k=1}^{i}\left(\int_{t_{k-1}}^{t_{k}}\left|f\left(s, x(s),\left(S_{q_{k-1}} x\right)(s)\right)\right| d_{q_{k-1}} s+\left|I_{k}^{*}\left(x\left(t_{k}\right)\right)\right|\right) \\
& +\left|\int_{t_{i}}^{\tau_{2}} \int_{t_{i}}^{s}\right| f\left(r, x(r),\left(S_{q_{i}} x\right)(r)\right)\left|d_{q_{i}} r d_{q_{i}} s-\int_{t_{i}}^{\tau_{1}} \int_{t_{i}}^{s}\right| f\left(r, x(r),\left(S_{q_{i}} x\right)(r)\right)\left|d_{q_{i}} r d_{q_{i}} s\right| \\
& \leq \frac{\left|\tau_{2}-\tau_{1}\right|}{T} \sum_{k=1}^{m}\left[\frac{N_{1}\left(t_{k}-t_{k-1}\right)^{2}}{1+q_{k-1}}+N_{2}\right] \\
& +\frac{\left|\tau_{2}-\tau_{1}\right|}{T} \sum_{k=1}^{m}\left[N_{1}\left(t_{k}-t_{k-1}\right)+N_{3}\right]\left(T-t_{k}+1\right)+\frac{\left|\tau_{2}-\tau_{1}\right|}{T}\left[\frac{N_{1}\left(T-t_{m}\right)^{2}}{1+q_{m}}\right] \\
& +\frac{\left|\tau_{2}-\tau_{1}\right|}{T}\left[N_{1}\left(T-t_{m}\right)\right]+\left|\tau_{2}-\tau_{1}\right| \sum_{k=1}^{i}\left[N_{1}\left(t_{k}-t_{k-1}\right)+N_{3}\right] \\
& +\frac{\left|\tau_{2}-\tau_{1}\right| N_{1}}{1+q_{i}}\left(\tau_{2}+\tau_{1}+2 t_{i}\right)
\end{aligned}
$$

As $\tau_{1} \rightarrow \tau_{2}$, the right-hand side of the above inequality (which is independent of $x$ ) tends to zero. As a consequence of Steps 1 to 3, together with the Arzelá-Ascoli theorem, we deduce that $\mathcal{A}: P C(J, \mathbb{R}) \rightarrow P C(J, \mathbb{R})$ is completely continuous.

Step 4: We show that the set

$$
E=\{x \in P C(J, \mathbb{R}): x=\theta \mathcal{A} x \text { for some } 0<\theta<1\}
$$

\section{is bounded.}

Let $x \in E$. Then $x(t)=\theta(\mathcal{A} x)(t)$ for some $0<\theta<1$. Thus, for each $t \in J$, by using the computations of Step 2, we have that

$$
\|\mathcal{A} x\| \leq \rho .
$$

This shows that the set $E$ is bounded. As a consequence of Schaefer's fixed point theorem, we conclude that $\mathcal{A}$ has a fixed point which is a solution of the impulsive $q_{k}$-integrodifference boundary value problem (1.1).

The third existence result for the impulsive boundary value problem (1.1) is based on Krasnoselskii's fixed point theorem.

Lemma 3.3 (Krasnoselskii's fixed point theorem) [19] Let M be a closed, bounded, convex and nonempty subset of a Banach space X. Let A, B be the operators such that

(a) $A x+B y \in M$ whenever $x, y \in M$;

(b) $A$ is a compact and continuous; 
(c) B is a contraction mapping.

Then there exists $z \in M$ such that $z=A z+B z$.

For convenience we put

$$
\begin{aligned}
\Lambda= & \frac{1+2 T}{T} \sum_{k=1}^{m+1} \frac{\left(t_{k}-t_{k-1}\right)^{2}}{1+q_{k-1}}+\frac{1+T}{T} \sum_{k=1}^{m+1}\left(t_{k}-t_{k-1}\right) \\
& +\frac{1+2 T}{T} \sum_{k=1}^{m}\left(T-t_{k}\right)\left(t_{k}-t_{k-1}\right),
\end{aligned}
$$

and

$$
\lambda_{1}=\frac{m(1+2 T) N_{1}}{T}+\frac{m(1+T) N_{2}}{T}+\frac{(1+2 T) N_{2}}{T} \sum_{k=1}^{m}\left(T-t_{k}\right) .
$$

Theorem 3.4 Let $f: J \times \mathbb{R} \rightarrow \mathbb{R}$ be a continuous function. Assume that:

$\left(\mathrm{A}_{1}\right)|f(t, x, y)| \leq \mu(t), \forall(t, x, y) \in J \times \mathbb{R} \times \mathbb{R}$ and $\mu \in C\left(J, \mathbb{R}^{+}\right)$.

$\left(\mathrm{A}_{2}\right)$ There exist constants $N_{1}, N_{2}>0$ such that $\left|I_{k}(x)\right| \leq N_{1}$ and $\left|I_{k}^{*}(x)\right| \leq N_{2}, \forall x \in \mathbb{R}$, for $k=1,2, \ldots, m$.

$\left(\mathrm{A}_{3}\right)$ There exist constants $K_{1}, K_{2}>0$ such that $\left|I_{k}(x)-I_{k}(y)\right| \leq K_{1}|x-y|$ and $\left|I_{k}^{*}(x)-I_{k}^{*}(y)\right| \leq$ $K_{2}|x-y|, \forall x, y \in \mathbb{R}$, for $k=1,2, \ldots, m$.

If

$$
\frac{m(1+2 T) K_{1}}{T}+\frac{m(1+T) K_{2}}{T}+\frac{(1+2 T) K_{2}}{T} \sum_{k=1}^{m}\left(T-t_{k}\right)<1
$$

then boundary value problem (1.1) has at least one solution on J.

Proof We define $\sup _{t \in J}|\mu(t)|=\|\mu\|$ and choose a suitable constant $\rho$ as

$$
\rho \geq\|\mu\| \Lambda+\lambda_{1}
$$

where $\Lambda$ and $\lambda_{1}$ are defined by (3.5) and (3.6), respectively. We define the operators $\Phi$ and $\Psi$ on $B_{\rho}=\{x \in P C(J, \mathbb{R}):\|x\| \leq \rho\}$ as

$$
\begin{aligned}
(\Phi x)(t)= & \left(\frac{1-t}{T}\right) \sum_{k=1}^{m}\left(\int_{t_{k-1}}^{t_{k}} \int_{t_{k-1}}^{s} f\left(r, x(r),\left(S_{q_{k-1}} x\right)(r)\right) d_{q_{k-1}} r d_{q_{k-1}} s\right) \\
& +\left(\frac{1-t}{T}\right) \sum_{k=1}^{m}\left(\int_{t_{k-1}}^{t_{k}} f\left(s, x(s),\left(S_{q_{k-1}} x\right)(s)\right) d_{q_{k-1}} s\right)\left(T-t_{k}+1\right) \\
& +\left(\frac{1-t}{T}\right) \int_{t_{m}}^{T} \int_{t_{m}}^{s} f\left(r, x(r),\left(S_{q_{m}} x\right)(r)\right) d_{q_{m}} r d_{q_{m}} s \\
& +\left(\frac{1-t}{T}\right) \int_{t_{m}}^{T} f\left(s, x(s),\left(S_{q_{m}} x\right)(s)\right) d_{q_{m}} s
\end{aligned}
$$




$$
\begin{aligned}
& +\sum_{0<t_{k}<t}\left(\int_{t_{k-1}}^{t_{k}} \int_{t_{k-1}}^{s} f\left(r, x(r),\left(S_{q_{k-1}} x\right)(r)\right) d_{q_{k-1}} r d_{q_{k-1}} s\right) \\
& +\sum_{0<t_{k}<t}\left(\int_{t_{k-1}}^{t_{k}} f\left(s, x(s),\left(S_{q_{k-1}} x\right)(s)\right) d_{q_{k-1}} s\right)\left(t-t_{k}\right) \\
& +\int_{t_{k}}^{t} \int_{t_{k}}^{s} f\left(r, x(r),\left(S_{q_{k}} x\right)(r)\right) d_{q_{k}} r d_{q_{k}} s
\end{aligned}
$$

and

$$
\begin{aligned}
(\Psi x)(t)= & \left(\frac{1-t}{T}\right) \sum_{k=1}^{m} I_{k}\left(x\left(t_{k}\right)\right)+\left(\frac{1-t}{T}\right) \sum_{k=1}^{m} I_{k}^{*}\left(x\left(t_{k}\right)\right)\left(T-t_{k}+1\right) \\
& +\sum_{0<t_{k}<t} I_{k}\left(x\left(t_{k}\right)\right)+\sum_{0<t_{k}<t} I_{k}^{*}\left(x\left(t_{k}\right)\right)\left(t-t_{k}\right) .
\end{aligned}
$$

For $x, y \in B_{\rho}$, we have

$$
\begin{aligned}
\|\Phi x+\Psi y\| \leq & \|\mu\|\left\{\frac{1+2 T}{T} \sum_{k=1}^{m+1} \frac{\left(t_{k}-t_{k-1}\right)^{2}}{1+q_{k-1}}+\frac{1+T}{T} \sum_{k=1}^{m+1}\left(t_{k}-t_{k-1}\right)\right. \\
& \left.+\frac{1+2 T}{T} \sum_{k=1}^{m}\left(T-t_{k}\right)\left(t_{k}-t_{k-1}\right)\right\} \\
& +\frac{m(1+2 T) N_{1}}{T}+\frac{m(1+T) N_{2}}{T}+\frac{(1+2 T) N_{2}}{T} \sum_{k=1}^{m}\left(T-t_{k}\right) \\
= & \|\mu\| \Lambda+\lambda_{1} \\
\leq & \rho .
\end{aligned}
$$

Thus, $\Phi x+\Psi y \in B_{\rho}$.

For $x, y \in P C(J, \mathbb{R})$, from $\left(\mathrm{A}_{3}\right)$, we have

$$
\begin{aligned}
\|\Psi x-\Psi y\| \leq & \frac{1+2 T}{T} \sum_{k=1}^{m}\left|I_{k}\left(x\left(t_{k}\right)\right)-I_{k}\left(y\left(t_{k}\right)\right)\right|+\frac{1+T}{T} \sum_{k=1}^{m}\left|I_{k}^{*}\left(x\left(t_{k}\right)\right)-I_{k}^{*}\left(y\left(t_{k}\right)\right)\right| \\
& +\frac{1+2 T}{T} \sum_{k=1}^{m}\left|I_{k}^{*}\left(x\left(t_{k}\right)\right)-I_{k}^{*}\left(y\left(t_{k}\right)\right)\right|\left(T-t_{k}\right) \\
\leq & \frac{m(1+2 T) K_{1}\|x-y\|}{T}+\frac{m(1+T) K_{2}\|x-y\|}{T} \\
& +\frac{(1+2 T) K_{2}\|x-y\|}{T} \sum_{k=1}^{m}\left(T-t_{k}\right),
\end{aligned}
$$

which implies, by (3.7), that $\Psi$ is a contraction mapping.

Continuity of $f$ implies that the operator $\Phi$ is continuous. Also, $\Phi$ is uniformly bounded on $B_{\rho}$ as

$$
\|\Phi x\| \leq\|\mu\| \Lambda
$$

Now we prove the compactness of the operator $\Phi$. 
We define $\sup _{(t, x) \in J \times B_{\rho}}|f(t, x)|=\bar{f}<\infty, \tau_{1}, \tau_{2} \in\left(t_{i}, t_{i+1}\right)$ for some $i \in\{0,1, \ldots, m\}$ with $\tau_{1}<$ $\tau_{2}$ and consequently we get

$$
\begin{aligned}
&\left|(\Phi x)\left(\tau_{2}\right)-(\Phi x)\left(\tau_{1}\right)\right| \leq \frac{\left|\tau_{2}-\tau_{1}\right|}{T} \sum_{k=1}^{m}\left(\int_{t_{k-1}}^{t_{k}} \int_{t_{k-1}}^{s}\left|f\left(r, x(r),\left(S_{q_{k-1}} x\right)(r)\right)\right| d_{q_{k-1}} r d_{q_{k-1}} s\right) \\
&+\frac{\left|\tau_{2}-\tau_{1}\right|}{T} \sum_{k=1}^{m}\left(\int_{t_{k-1}}^{t_{k}}\left|f\left(s, x(s),\left(S_{q_{k-1}} x\right)(s)\right)\right| d_{q_{k-1}} s\right)\left(T-t_{k}+1\right) \\
&+\frac{\left|\tau_{2}-\tau_{1}\right|}{T} \int_{t_{m}}^{T} \int_{t_{m}}^{s}\left|f\left(r, x(r),\left(S_{q_{m}} x\right)(r)\right)\right| d_{q_{m}} r d_{q_{m}} s \\
&+\frac{\left|\tau_{2}-\tau_{1}\right|}{T} \int_{t_{m}}^{T}\left|f\left(s, x(s),\left(S_{q_{m}} x\right)(s)\right)\right| d_{q_{m}} s \\
&+\left|\tau_{2}-\tau_{1}\right| \sum_{k=1}^{i}\left(\int_{t_{k-1}}^{t_{k}}\left|f\left(s, x(s),\left(S_{q_{k-1}} x\right)(s)\right)\right| d_{q_{k-1}} s\right) \\
&+\left|\int_{t_{i}}^{\tau_{2}} \int_{t_{i}}^{s}\right| f\left(r, x(r),\left(S_{q_{i}} x\right)(r)\right)\left|d_{q_{i}} r d_{q_{i}} s-\int_{t_{i}}^{\tau_{1}} \int_{t_{i}}^{s}\right| f\left(r, x(r),\left(S_{q_{i}} x\right)(r)\right)\left|d_{q_{i}} r d_{q_{i}} s\right| \\
& \leq \frac{\left|\tau_{2}-\tau_{1}\right| \bar{f}}{T} \sum_{k=1}^{m} \frac{\left(t_{k}-t_{k-1}\right)^{2}}{1+q_{k-1}}+\frac{\left|\tau_{2}-\tau_{1}\right| \bar{f}}{T} \sum_{k=1}^{m}\left(t_{k}-t_{k-1}\right)\left(T-t_{k}+1\right) \\
&+\frac{\left|\tau_{2}-\tau_{1}\right| \bar{f}}{T} \frac{\left(T-t_{m}\right)^{2}}{1+q_{m}}+\frac{\left|\tau_{2}-\tau_{1}\right| \bar{f}}{T}\left(T-t_{m}\right)+\left|\tau_{2}-\tau_{1}\right| \bar{f} \sum_{k=1}^{i}\left(t_{k}-t_{k-1}\right) \\
&+\frac{\left|\tau_{2}-\tau_{1}\right| \bar{f}}{1+q_{i}}\left(\tau_{2}+\tau_{1}+2 t_{i}\right), \\
& \hline
\end{aligned}
$$

which is independent of $x$ and tends to zero as $\tau_{2}-\tau_{1} \rightarrow 0$. Thus, $\Phi$ is equicontinuous. So $\Phi$ is relatively compact on $B_{\rho}$. Hence, by the Arzelá-Ascoli theorem, $\Phi$ is compact on $B_{\rho}$. Thus all the assumptions of Lemma 3.3 are satisfied. So the boundary value problem (1.1) has at least one solution on $J$. The proof is completed.

Our final, fourth existence result is based on the Leray-Schauder Nonlinear Alternative.

Lemma 3.5 (Nonlinear alternative for single valued maps) [20] Let E be a Banach space, $C$ a closed, convex subset of $E, U$ an open subset of $C$ and $0 \in U$. Suppose that $F: \bar{U} \rightarrow C$ is a continuous, compact (that is, $F(\bar{U})$ is a relatively compact subset of $C$ ) map. Then either

(i) F has a fixed point in $\bar{U}$, or

(ii) there is a $u \in \partial U$ (the boundary of $U$ in $C$ ) and $\theta \in(0,1)$ with $u=\theta F(u)$.

\section{Theorem 3.6 Assume that:}

$\left(\mathrm{A}_{4}\right)$ There exist a continuous nondecreasing function $\psi:[0, \infty) \rightarrow(0, \infty)$ and a continuous function $p: J \rightarrow \mathbb{R}^{+}$such that

$$
|f(t, x, y)| \leq p(t) \psi(|x|)+|y| \quad \text { for each }(t, x, y) \in J \times \mathbb{R} \times \mathbb{R} .
$$


$\left(\mathrm{A}_{5}\right)$ There exist continuous nondecreasing functions $\varphi_{1}, \varphi_{2}:[0, \infty) \rightarrow(0, \infty)$ such that

$$
\left|I_{k}(x)\right| \leq \varphi_{1}(|x|) \text { and }\left|I_{k}^{*}(x)\right| \leq \varphi_{2}(|x|)
$$

for all $x \in \mathbb{R}, k=1,2, \ldots, m$.

$\left(\mathrm{A}_{6}\right)$ There exists a constant $M^{*}>0$ such that

$$
\frac{M^{*}}{p_{0} \psi\left(M^{*}\right) Q_{1}+\phi_{0} M^{*} Q_{2}+Q_{3}}>1
$$

where $p_{0}=\max \{p(t): t \in J\}, \phi_{0}=\max \{\phi(t, s):(t, s) \in J \times J\}$ and

$$
\begin{aligned}
Q_{1}= & \frac{1+2 T}{T} \sum_{k=1}^{m+1} \frac{\left(t_{k}-t_{k-1}\right)^{2}}{1+q_{k-1}}+\frac{1+T}{T} \sum_{k=1}^{m+1}\left(t_{k}-t_{k-1}\right) \\
& +\frac{1+2 T}{T} \sum_{k=1}^{m}\left(T-t_{k}\right)\left(t_{k}-t_{k-1}\right), \\
Q_{2}= & \frac{1+2 T}{T} \sum_{k=1}^{m+1} \frac{\left(t_{k}-t_{k-1}\right)^{3}}{1+q_{k-1}+q_{k-1}^{2}}+\frac{1+T}{T} \sum_{k=1}^{m+1} \frac{\left(t_{k}-t_{k-1}\right)^{2}}{1+q_{k-1}} \\
& +\frac{1+2 T}{T} \sum_{k=1}^{m}\left(T-t_{k}\right) \frac{\left(t_{k}-t_{k-1}\right)^{2}}{1+q_{k-1}}, \\
Q_{3}= & \frac{m(1+2 T) \varphi_{1}\left(M^{*}\right)}{T}+\frac{m(1+T) \varphi_{2}\left(M^{*}\right)}{T}+\frac{(1+2 T) \varphi_{2}\left(M^{*}\right)}{T} \sum_{k=1}^{m}\left(T-t_{k}\right) .
\end{aligned}
$$

Then the impulsive boundary value problem (1.1) has at least one solution on J.

Proof First we show that $\mathcal{A}$ maps bounded sets (balls) into bounded sets in $P C(J, \mathbb{R})$. For a positive number $\bar{\rho}$, let $B_{\bar{\rho}}=\{x \in P C(J, \mathbb{R}):\|x\| \leq \bar{\rho}\}$ be a bounded ball in $P C(J, \mathbb{R})$. Then for $t \in J$ we have

$$
\begin{aligned}
& |(\mathcal{A} x)(t)| \\
& \leq \frac{|1-t|}{T} \sum_{k=1}^{m}\left(\int_{t_{k-1}}^{t_{k}} \int_{t_{k-1}}^{s}\left|f\left(r, x(r),\left(S_{q_{k-1}} x\right)(r)\right)\right| d_{q_{k-1}} r d_{q_{k-1}} s+\left|I_{k}\left(x\left(t_{k}\right)\right)\right|\right) \\
& \quad+\frac{|1-t|}{T} \sum_{k=1}^{m}\left(\int_{t_{k-1}}^{t_{k}}\left|f\left(s, x(s),\left(S_{q_{k-1}} x\right)(s)\right)\right| d_{q_{k-1}} s+\left|I_{k}^{*}\left(x\left(t_{k}\right)\right)\right|\right)\left(T-t_{k}+1\right) \\
& \quad+\frac{|1-t|}{T} \int_{t_{m}}^{T} \int_{t_{m}}^{s}\left|f\left(r, x(r),\left(S_{q_{m}} x\right)(r)\right)\right| d_{q_{m}} r d_{q_{m}} s \\
& \quad+\frac{|1-t|}{T} \int_{t_{m}}^{T}\left|f\left(s, x(s),\left(S_{q_{m}} x\right)(s)\right)\right| d_{q_{m}} s \\
& \quad+\sum_{k=1}^{m}\left(\int_{t_{k-1}}^{t_{k}} \int_{t_{k-1}}^{s}\left|f\left(r, x(r),\left(S_{q_{k-1}} x\right)(r)\right)\right| d_{q_{k-1}} r d_{q_{k-1}} s+\left|I_{k}\left(x\left(t_{k}\right)\right)\right|\right) \\
& \quad+\sum_{k=1}^{m}\left(\int_{t_{k-1}}^{t_{k}}\left|f\left(s, x(s),\left(S_{q_{k-1}} x\right)(s)\right)\right| d_{q_{k-1}} s+\left|I_{k}^{*}\left(x\left(t_{k}\right)\right)\right|\right)\left(T-t_{k}\right)
\end{aligned}
$$


Thaiprayoon et al. Advances in Difference Equations 2014, 2014:88

Page 17 of 23

http://www.advancesindifferenceequations.com/content/2014/1/88

$$
\begin{aligned}
& +\int_{t_{m}}^{T} \int_{t_{m}}^{s}\left|f\left(r, x(r),\left(S_{q_{m}} x\right)(r)\right)\right| d_{q_{m}} r d_{q_{m}} s \\
& \leq \frac{1+2 T}{T} \sum_{k=1}^{m+1} \int_{t_{k-1}}^{t_{k}} \int_{t_{k-1}}^{s}\left(p_{0} \psi(\|x\|)+\phi_{0}\|x\| \int_{t_{k-1}}^{r} d_{q_{k-1}} u\right) d_{q_{k-1}} r d_{q_{k-1}} s \\
& +\frac{1+T}{T} \sum_{k=1}^{m+1} \int_{t_{k-1}}^{t_{k}}\left(p_{0} \psi(\|x\|)+\phi_{0}\|x\| \int_{t_{k-1}}^{s} d_{q_{k-1}} r\right) d_{q_{k-1}} s \\
& +\frac{1+2 T}{T} \sum_{k=1}^{m}\left(T-t_{k}\right) \int_{t_{k-1}}^{t_{k}}\left(p_{0} \psi(\|x\|)+\phi_{0}\|x\| \int_{t_{k-1}}^{s} d_{q_{k-1}} r\right) d_{q_{k-1}} s \\
& +\frac{1+2 T}{T} \sum_{k=1}^{m} \varphi_{1}(\|x\|)+\frac{1+T}{T} \sum_{k=1}^{m} \varphi_{2}(\|x\|)+\frac{1+2 T}{T} \sum_{k=1}^{m} \varphi_{2}(\|x\|)\left(T-t_{k}\right) \\
& \leq \frac{1+2 T}{T} \sum_{k=1}^{m+1}\left[\frac{p_{0} \psi(\|x\|)\left(t_{k}-t_{k-1}\right)^{2}}{1+q_{k-1}}+\frac{\phi_{0}\|x\|\left(t_{k}-t_{k-1}\right)^{3}}{1+q_{k-1}+q_{k-1}^{2}}\right] \\
& +\frac{1+T}{T} \sum_{k=1}^{m+1}\left[p_{0} \psi(\|x\|)\left(t_{k}-t_{k-1}\right)+\frac{\phi_{0}\|x\|\left(t_{k}-t_{k-1}\right)^{2}}{1+q_{k-1}}\right] \\
& +\frac{1+2 T}{T} \sum_{k=1}^{m}\left(T-t_{k}\right)\left[p_{0} \psi(\|x\|)\left(t_{k}-t_{k-1}\right)+\frac{\phi_{0}\|x\|\left(t_{k}-t_{k-1}\right)^{2}}{1+q_{k-1}}\right] \\
& +\frac{m(1+2 T) \varphi_{1}(\|x\|)}{T}+\frac{m(1+T) \varphi_{2}(\|x\|)}{T} \\
& +\frac{(1+2 T) \varphi_{2}(\|x\|)}{T} \sum_{k=1}^{m}\left(T-t_{k}\right) \\
& \leq \frac{1+2 T}{T} \sum_{k=1}^{m+1}\left[\frac{p_{0} \psi(\bar{\rho})\left(t_{k}-t_{k-1}\right)^{2}}{1+q_{k-1}}+\frac{\phi_{0} \bar{\rho}\left(t_{k}-t_{k-1}\right)^{3}}{1+q_{k-1}+q_{k-1}^{2}}\right] \\
& +\frac{1+T}{T} \sum_{k=1}^{m+1}\left[p_{0} \psi(\bar{\rho})\left(t_{k}-t_{k-1}\right)+\frac{\phi_{0} \bar{\rho}\left(t_{k}-t_{k-1}\right)^{2}}{1+q_{k-1}}\right] \\
& +\frac{1+2 T}{T} \sum_{k=1}^{m}\left(T-t_{k}\right)\left[p_{0} \psi(\bar{\rho})\left(t_{k}-t_{k-1}\right)+\frac{\phi_{0} \bar{\rho}\left(t_{k}-t_{k-1}\right)^{2}}{1+q_{k-1}}\right] \\
& +\frac{m(1+2 T) \varphi_{1}(\bar{\rho})}{T}+\frac{m(1+T) \varphi_{2}(\bar{\rho})}{T}+\frac{(1+2 T) \varphi_{2}(\bar{\rho})}{T} \sum_{k=1}^{m}\left(T-t_{k}\right) \\
& :=K \text {. }
\end{aligned}
$$

Hence, we deduce that $\|\mathcal{A} x\| \leq K$.

Next we show that $\mathcal{A}$ maps bounded sets into equicontinuous sets of $P C(J, \mathbb{R})$.

Let $\tau_{1}, \tau_{2} \in J_{i}$ for some $i \in\{0,1,2, \ldots, m\}, \tau_{1}<\tau_{2}, B_{\bar{\rho}}$ be a bounded set of $P C(J, \mathbb{R})$ as in the previous step, and let $x \in B_{\bar{\rho}}$. Then we have

$$
\begin{aligned}
& \left|(\mathcal{A} x)\left(\tau_{2}\right)-(\mathcal{A} x)\left(\tau_{1}\right)\right| \\
& \quad \leq \frac{\left|\tau_{2}-\tau_{1}\right|}{T} \sum_{k=1}^{m}\left(\int_{t_{k-1}}^{t_{k}} \int_{t_{k-1}}^{s}\left|f\left(r, x(r),\left(S_{q_{k-1}} x\right)(r)\right)\right| d_{q_{k-1}} r d_{q_{k-1}} s+\left|I_{k}\left(x\left(t_{k}\right)\right)\right|\right)
\end{aligned}
$$




$$
\begin{aligned}
& +\frac{\left|\tau_{2}-\tau_{1}\right|}{T} \sum_{k=1}^{m}\left(\int_{t_{k-1}}^{t_{k}}\left|f\left(s, x(s),\left(S_{q_{k-1}} x\right)(s)\right)\right| d_{q_{k-1}} s+\left|I_{k}^{*}\left(x\left(t_{k}\right)\right)\right|\right)\left(T-t_{k}+1\right) \\
& +\frac{\left|\tau_{2}-\tau_{1}\right|}{T} \int_{t_{m}}^{T} \int_{t_{m}}^{s}\left|f\left(r, x(r),\left(S_{q_{m}} x\right)(r)\right)\right| d_{q_{m}} r d_{q_{m}} s \\
& +\frac{\left|\tau_{2}-\tau_{1}\right|}{T} \int_{t_{m}}^{T}\left|f\left(s, x(s),\left(S_{q_{m}} x\right)(s)\right)\right| d_{q_{m}} s \\
& +\left|\tau_{2}-\tau_{1}\right| \sum_{k=1}^{i}\left(\int_{t_{k-1}}^{t_{k}}\left|f\left(s, x(s),\left(S_{q_{k-1}} x\right)(s)\right)\right| d_{q_{k-1}} s+\left|I_{k}^{*}\left(x\left(t_{k}\right)\right)\right|\right) \\
& +\left|\int_{t_{i}}^{\tau_{2}} \int_{t_{i}}^{s}\right| f\left(r, x(r),\left(S_{q_{i}} x\right)(r)\right)\left|d_{q_{i}} r d_{q_{i}} s-\int_{t_{i}}^{\tau_{1}} \int_{t_{i}}^{s}\right| f\left(r, x(r),\left(S_{q_{i}} x\right)(r)\right)\left|d_{q_{i}} r d_{q_{i}} s\right| \\
& \leq \frac{\left|\tau_{2}-\tau_{1}\right|}{T} \sum_{k=1}^{m}\left[\frac{p_{0} \psi(\bar{\rho})\left(t_{k}-t_{k-1}\right)^{2}}{1+q_{k-1}}+\frac{\phi_{0} \bar{\rho}\left(t_{k}-t_{k-1}\right)^{3}}{1+q_{k-1}+q_{k-1}^{2}}+\varphi_{1}(\bar{\rho})\right] \\
& +\frac{\left|\tau_{2}-\tau_{1}\right|}{T} \sum_{k=1}^{m}\left[p_{0} \psi(\bar{\rho})\left(t_{k}-t_{k-1}\right)+\frac{\phi_{0} \bar{\rho}\left(t_{k}-t_{k-1}\right)^{2}}{1+q_{k-1}}+\varphi_{2}(\bar{\rho})\right]\left(T-t_{k}+1\right) \\
& +\frac{\left|\tau_{2}-\tau_{1}\right|}{T}\left[\frac{p_{0} \psi(\bar{\rho})\left(T-t_{m}\right)^{2}}{1+q_{m}}+\frac{\phi_{0} \bar{\rho}\left(T-t_{m}\right)^{3}}{1+q_{m}+q_{m}^{2}}\right] \\
& +\frac{\left|\tau_{2}-\tau_{1}\right|}{T}\left[p_{0} \psi(\bar{\rho})\left(T-t_{m}\right)+\frac{\phi_{0} \bar{\rho}\left(T-t_{m}\right)^{2}}{1+q_{m}}\right] \\
& +\left|\tau_{2}-\tau_{1}\right| \sum_{k=1}^{i}\left[p_{0} \psi(\bar{\rho})\left(t_{k}-t_{k-1}\right)+\frac{\phi_{0} \bar{\rho}\left(t_{k}-t_{k-1}\right)^{2}}{1+q_{k-1}}+\varphi_{2}(\bar{\rho})\right] \\
& +\frac{\left|\tau_{2}-\tau_{1}\right| p_{0} \psi(\bar{\rho})}{1+q_{i}}\left(\tau_{2}+\tau_{1}+2 t_{i}\right) \\
& +\frac{\left|\tau_{2}-\tau_{1}\right| \phi_{0} \bar{\rho}}{1+q_{i}+q_{i}^{2}}\left(\tau_{2}^{2}+\tau_{2} \tau_{1}+\tau_{1}^{2}+3\left(\tau_{2}+\tau_{1}\right) t_{i}+3 t_{i}^{2}\right) .
\end{aligned}
$$

The right-hand side of the above inequality, which is independent of $x$, tends to zero as $\tau_{1} \rightarrow \tau_{2}$. From all above and by the Arzelá-Ascoli theorem $\mathcal{A}: P C(J, \mathbb{R}) \rightarrow P C(J, \mathbb{R})$ is completely continuous.

The result will follow from the Leray-Schauder nonlinear alternative (Lemma 3.5) once we have proved the boundedness of the set of all solutions to the equations $x(t)=\theta(\mathcal{A} x)(t)$ for some $0<\theta<1$.

Let $x$ be a solution. Thus, for each $t \in J$, we have

$(\mathcal{A} x)(t)$

$$
\begin{aligned}
= & \theta\left(\frac{1-t}{T}\right) \sum_{k=1}^{m}\left(\int_{t_{k-1}}^{t_{k}} \int_{t_{k-1}}^{s} f\left(r, x(r),\left(S_{q_{k-1}} x\right)(r)\right) d_{q_{k-1}} r d_{q_{k-1}} s+I_{k}\left(x\left(t_{k}\right)\right)\right) \\
& +\theta\left(\frac{1-t}{T}\right) \sum_{k=1}^{m}\left(\int_{t_{k-1}}^{t_{k}} f\left(s, x(s),\left(S_{q_{k-1}} x\right)(s)\right) d_{q_{k-1}} s+I_{k}^{*}\left(x\left(t_{k}\right)\right)\right)\left(T-t_{k}+1\right) \\
& +\theta\left(\frac{1-t}{T}\right) \int_{t_{m}}^{T} \int_{t_{m}}^{s} f\left(r, x(r),\left(S_{q_{m}} x\right)(r)\right) d_{q_{m}} r d_{q_{m}} s
\end{aligned}
$$


Thaiprayoon et al. Advances in Difference Equations 2014, 2014:88

Page 19 of 23

http://www.advancesindifferenceequations.com/content/2014/1/88

$$
\begin{aligned}
& +\theta\left(\frac{1-t}{T}\right) \int_{t_{m}}^{T} f\left(s, x(s),\left(S_{q_{m}} x\right)(s)\right) d_{q_{m}} s \\
& +\theta \sum_{0<t_{k}<t}\left(\int_{t_{k-1}}^{t_{k}} \int_{t_{k-1}}^{s} f\left(r, x(r),\left(S_{q_{k-1}} x\right)(r)\right) d_{q_{k-1}} r d_{q_{k-1}} s+I_{k}\left(x\left(t_{k}\right)\right)\right) \\
& +\theta \sum_{0<t_{k}<t}\left(\int_{t_{k-1}}^{t_{k}} f\left(s, x(s),\left(S_{q_{k-1}} x\right)(s)\right) d_{q_{k-1}} s+I_{k}^{*}\left(x\left(t_{k}\right)\right)\right)\left(t-t_{k}\right) \\
& +\theta \int_{t_{k}}^{t} \int_{t_{k}}^{s} f\left(r, x(r),\left(S_{q_{k}} x\right)(r)\right) d_{q_{k}} r d_{q_{k}} s .
\end{aligned}
$$

This implies by $\left(\mathrm{A}_{4}\right)$ and $\left(\mathrm{A}_{5}\right)$ that for each $t \in J$, we have

$$
\begin{aligned}
& |(\mathcal{A} x)(t)| \leq \frac{\theta(1+2 T)}{T} \sum_{k=1}^{m+1} \int_{t_{k-1}}^{t_{k}} \int_{t_{k-1}}^{s}\left(p(t) \psi(|x|)+\left|S_{q_{k-1}} x\right|\right) d_{q_{k-1}} r d_{q_{k-1}} s \\
& +\frac{\theta(1+T)}{T} \sum_{k=1}^{m+1} \int_{t_{k-1}}^{t_{k}}\left(p(t) \psi(|x|)+\left|S_{q_{k-1}} x\right|\right) d_{q_{k-1}} s \\
& +\frac{\theta(1+2 T)}{T} \sum_{k=1}^{m}\left(T-t_{k}\right) \int_{t_{k-1}}^{t_{k}}\left(p(t) \psi(|x|)+\left|S_{q_{k-1}} x\right|\right) d_{q_{k-1}} s \\
& +\frac{\theta(1+2 T)}{T} \sum_{k=1}^{m} \varphi_{1}(|x|)+\frac{\theta(1+T)}{T} \sum_{k=1}^{m} \varphi_{2}(|x|) \\
& +\frac{\theta(1+2 T)}{T} \sum_{k=1}^{m} \varphi_{2}(|x|)\left(T-t_{k}\right) \\
& \leq \frac{1+2 T}{T} \sum_{k=1}^{m+1} \int_{t_{k-1}}^{t_{k}} \int_{t_{k-1}}^{s}\left(p_{0} \psi(\|x\|)+\phi_{0}\|x\| \int_{t_{k-1}}^{r} d_{q_{k-1}} u\right) d_{q_{k-1}} r d_{q_{k-1}} s \\
& +\frac{1+T}{T} \sum_{k=1}^{m+1} \int_{t_{k-1}}^{t_{k}}\left(p_{0} \psi(\|x\|)+\phi_{0}\|x\| \int_{t_{k-1}}^{s} d_{q_{k-1}} r\right) d_{q_{k-1}} s \\
& +\frac{1+2 T}{T} \sum_{k=1}^{m}\left(T-t_{k}\right) \int_{t_{k-1}}^{t_{k}}\left(p_{0} \psi(\|x\|)+\phi_{0}\|x\| \int_{t_{k-1}}^{s} d_{q_{k-1}} r\right) d_{q_{k-1}} s \\
& +\frac{1+2 T}{T} \sum_{k=1}^{m} \varphi_{1}(\|x\|)+\frac{1+T}{T} \sum_{k=1}^{m} \varphi_{2}(\|x\|) \\
& +\frac{1+2 T}{T} \sum_{k=1}^{m} \varphi_{2}(\|x\|)\left(T-t_{k}\right) \\
& \leq \frac{1+2 T}{T} \sum_{k=1}^{m+1}\left[\frac{p_{0} \psi(\|x\|)\left(t_{k}-t_{k-1}\right)^{2}}{1+q_{k-1}}+\frac{\phi_{0}\|x\|\left(t_{k}-t_{k-1}\right)^{3}}{1+q_{k-1}+q_{k-1}^{2}}\right] \\
& +\frac{1+T}{T} \sum_{k=1}^{m+1}\left[p_{0} \psi(\|x\|)\left(t_{k}-t_{k-1}\right)+\frac{\phi_{0}\|x\|\left(t_{k}-t_{k-1}\right)^{2}}{1+q_{k-1}}\right] \\
& +\frac{1+2 T}{T} \sum_{k=1}^{m}\left(T-t_{k}\right)\left[p_{0} \psi(\|x\|)\left(t_{k}-t_{k-1}\right)+\frac{\phi_{0}\|x\|\left(t_{k}-t_{k-1}\right)^{2}}{1+q_{k-1}}\right]
\end{aligned}
$$




$$
\begin{aligned}
& +\frac{m(1+2 T) \varphi_{1}(\|x\|)}{T}+\frac{m(1+T) \varphi_{2}(\|x\|)}{T} \\
& +\frac{(1+2 T) \varphi_{2}(\|x\|)}{T} \sum_{k=1}^{m}\left(T-t_{k}\right) \\
& =p_{0} \psi(\|x\|)\left[\frac{1+2 T}{T} \sum_{k=1}^{m+1} \frac{\left(t_{k}-t_{k-1}\right)^{2}}{1+q_{k-1}}+\frac{1+T}{T} \sum_{k=1}^{m+1}\left(t_{k}-t_{k-1}\right)\right. \\
& \left.+\frac{1+2 T}{T} \sum_{k=1}^{m}\left(T-t_{k}\right)\left(t_{k}-t_{k-1}\right)\right] \\
& +\phi_{0}\|x\|\left[\frac{1+2 T}{T} \sum_{k=1}^{m+1} \frac{\left(t_{k}-t_{k-1}\right)^{3}}{1+q_{k-1}+q_{k-1}^{2}}+\frac{1+T}{T} \sum_{k=1}^{m+1} \frac{\left(t_{k}-t_{k-1}\right)^{2}}{1+q_{k-1}}\right. \\
& \left.+\frac{1+2 T}{T} \sum_{k=1}^{m}\left(T-t_{k}\right) \frac{\left(t_{k}-t_{k-1}\right)^{2}}{1+q_{k-1}}\right]+\frac{m(1+2 T) \varphi_{1}(\|x\|)}{T} \\
& +\frac{m(1+T) \varphi_{2}(\|x\|)}{T}+\frac{(1+2 T) \varphi_{2}(\|x\|)}{T} \sum_{k=1}^{m}\left(T-t_{k}\right) \\
& =p_{0} \psi(\|x\|) Q_{1}+\phi_{0}\|x\| Q_{2}+Q_{3} .
\end{aligned}
$$

Consequently, we have

$$
\frac{\|x\|}{p_{0} \psi(\|x\|) Q_{1}+\phi_{0}\|x\| Q_{2}+Q_{3}} \leq 1 .
$$

In view of $\left(\mathrm{A}_{6}\right)$, there exists $M^{*}$ such that $\|x\| \neq M^{*}$. Let us set

$$
U=\left\{x \in P C(J, \mathbb{R}):\|x\|<M^{*}\right\}
$$

Note that the operator $\mathcal{A}: \bar{U} \rightarrow P C(J, \mathbb{R})$ is continuous and completely continuous. From the choice of $U$, there is no $x \in \partial U$ such that $x=\theta \mathcal{A} x$ for some $\theta \in(0,1)$. Consequently, by the nonlinear alternative of Leray-Schauder type (Lemma 3.5), we deduce that $\mathcal{A}$ has a fixed point $x \in \bar{U}$ which is a solution of the problem (1.1). This completes the proof.

\section{Examples}

In this section, we will give examples to illustrate our main results.

Example 4.1 Consider the following boundary value problem for the nonlinear secondorder impulsive $q_{k}$-integro-difference equation:

$$
\left\{\begin{array}{l}
D_{\frac{k+1}{k+4}}^{2} x(t)=\frac{t}{e^{t}(t+3)^{2}} \frac{|x(t)|}{|x(t)|+3}+\frac{t^{2}}{36} \int_{t_{k}}^{t} \frac{\sin \pi(t-s)}{3} x(s) d_{q_{k}} s, \quad t \in J, t \neq t_{k}, \\
\Delta x\left(t_{k}\right)=\frac{\left|x\left(t_{k}\right)\right|}{9(k+8)+\left|x\left(t_{k}\right)\right|}, \quad k=1,2, \ldots, 9, \\
D_{\frac{k+1}{k+4}} x\left(t_{k}^{+}\right)-D_{\frac{k}{k+3}} x\left(t_{k}\right)=\frac{\left|x\left(t_{k}\right)\right|}{8(k+7)+\left|x\left(t_{k}\right)\right|}, \quad k=1,2, \ldots, 9, \\
x(0)+D_{\frac{1}{4}} x(0)=0, \quad x(1)+D_{\frac{10}{13}} x(1)=0 .
\end{array}\right.
$$

Here $J=[0,1], t_{k}=k / 10, q_{k}=(k+1) /(k+4)$ for $k=0,1,2, \ldots, 9, m=9, T=1, f\left(t, x, S_{q_{k}} x\right)=$ $(t|x|) /\left(e^{t}(t+3)^{2}(|x|+3)\right)+\left(t^{2} / 36\right) \int_{t_{k}}^{t}((\sin \pi(t-s)) / 3) x(s) d_{q_{k}} s, I_{k}(x)=|x| /(9(k+8)+|x|)$ and 
$I_{k}^{*}(x)=|x| /(8(k+7)+|x|)$. Since

$$
\begin{aligned}
& |f(t, x)-f(t, y)| \leq \frac{1}{27}|x-y|+(1 / 36)\left(S_{q_{k}}|x-y|\right), \\
& \left|I_{k}(x)-I_{k}(y)\right| \leq \frac{1}{81}|x-y| \text { and }\left|I_{k}^{*}(x)-I_{k}^{*}(y)\right| \leq \frac{1}{64}|x-y|,
\end{aligned}
$$

$\left(\mathrm{H}_{1}\right)$ and $\left(\mathrm{H}_{2}\right)$ are satisfied with $L_{1}=(1 / 27), L_{2}=(1 / 36), L_{3}=(1 / 81), L_{4}=(1 / 64)$. We can show that

$$
\omega \approx 0.9587977316<1 .
$$

Hence, by Theorem 3.1, boundary value problem (4.1) has a unique solution on $[0,1]$.

Example 4.2 Consider the following boundary value problem for the nonlinear secondorder impulsive $q_{k}$-integro-difference equation:

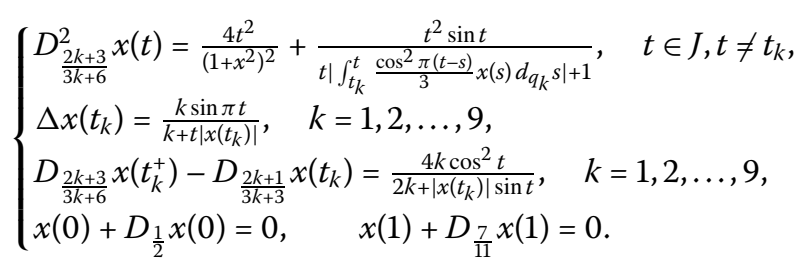

Here $J=[0,1], t_{k}=k / 10, q_{k}=(2 k+3) /(3 k+6)$ for $k=0,1,2, \ldots, 9, m=9, T=1, f\left(t, x, S_{q_{k}} x\right)=$ $\left(\left(4 t^{2}\right) /\left(1+x^{2}\right)^{2}\right)+\left(t^{2} \sin t\right) /\left(t\left|\int_{t_{k}}^{t}\left(\left(\cos ^{2} \pi(t-s)\right) / 3\right) x(s) d_{q_{k}} s\right|+1\right), I_{k}(x)=(k \sin \pi t) /\left(k+t\left|x\left(t_{k}\right)\right|\right)$ and $I_{k}^{*}(x)=\left(4 k \cos ^{2} t\right) /\left(2 k+\left|x\left(t_{k}\right)\right| \sin t\right)$. We can show that

$$
\begin{aligned}
& \left|f\left(t, x,\left(S_{q_{k}} x\right)\right)\right|=\left|\frac{4 t^{2}}{\left(1+x^{2}\right)^{2}}+\frac{t^{2} \sin t}{t\left|\int_{t_{k}}^{t} \frac{\cos ^{2} \pi(t-s)}{3} x(s) d_{q_{k}} s\right|+1}\right| \leq 5=N_{1}, \\
& \left|I_{k}(x)\right|=\left|\frac{k \sin \pi t}{k+t\left|x\left(t_{k}\right)\right|}\right| \leq 1=N_{2}, \quad \text { and } \quad\left|I_{k}^{*}(x)\right|=\left|\frac{4 k \cos ^{2} t}{2 k+\left|x\left(t_{k}\right)\right| \sin t}\right| \leq 2=N_{3} .
\end{aligned}
$$

Hence, by Theorem 3.2, boundary value problem (4.2) has at least one solution on $[0,1]$.

Example 4.3 Consider the following nonlinear second-order impulsive $q_{k}$-difference equation with separated boundary condition:

$$
\left\{\begin{array}{l}
D_{\frac{k+1}{3 k+2}}^{2} x(t)=\frac{t \sin \pi t}{(t+1)^{2}} \frac{|x|}{2|x|+1}+\frac{\cos \frac{\pi t^{2}}{2}}{\left|\int_{t_{k}}^{t} \frac{e^{t-s}}{t+e^{-s}} x(s) d_{q_{k} s}\right|}, \quad t \in J, t \neq t_{k}, \\
\Delta x\left(t_{k}\right)=\frac{\mid x\left(t_{k} \mid\right.}{7(k+8)+\left|x\left(t_{k}\right)\right|}, \quad k=1,2, \ldots, 9, \\
D_{\frac{k+1}{3 k+2}} x\left(t_{k}^{+}\right)-D_{\frac{k}{3 k-1}} x\left(t_{k}\right)=\frac{\left|x\left(t_{k}\right)\right|}{8(k+7)+\left|x\left(t_{k}\right)\right|}, \quad k=1,2, \ldots, 9, \\
x(0)+D_{\frac{1}{2}} x(0)=0, \quad x(1)+D_{\frac{10}{29}} x(1)=0,
\end{array}\right.
$$

where $J=[0,1], t_{k}=k / 10, q_{k}=(k+1) /(3 k+2)$ for $k=0,1,2, \ldots, 9, m=9, T=1$, $f(t, x)=\left(t \sin \pi t /(t+1)^{2}\right)(|x| /(2|x|+1))+\left(\cos \left(\pi t^{2} / 2\right)\right) /\left(\left|\int_{t_{k}}^{t}\left(e^{t-s} /\left(t+e^{t-s}\right)\right) x(s) d_{q_{k}} s\right|\right), I_{k}(x)=$ $\left|x\left(t_{k}\right)\right| /\left(7(k+8)+\left|x\left(t_{k}\right)\right|\right)$ and $I_{k}^{*}(x)=\left|x\left(t_{k}\right)\right| /\left(8(k+7)+\left|x\left(t_{k}\right)\right|\right)$. Since

$$
\left|I_{k}(x)-I_{k}(y)\right| \leq(1 / 63)|x-y| \quad \text { and } \quad\left|I_{k}^{*}(x)-I_{k}^{*}(y)\right| \leq(1 / 64)|x-y|
$$


$\left(\mathrm{A}_{3}\right)$ is satisfied with $L_{1}=(1 / 63), L_{2}=(1 / 64)$. It is easy to verify that $|f(t, x)| \leq \mu(t) \equiv$ $(t \sin \pi t) /(t+1)^{2}+e^{-t} \cos \left(\pi t^{2} / 2\right), I_{k}(x) \leq N_{1}=1$, and $I_{k}^{*}(x) \leq N_{2}=1$ for all $t \in[0,1], x \in \mathbb{R}$, $k=1, \ldots, m$. Thus $\left(\mathrm{A}_{1}\right)$ and $\left(\mathrm{A}_{2}\right)$ are satisfied. We can show that

$$
\frac{m(1+2 T) K_{1}}{T}+\frac{m(1+T) K_{2}}{T}+\frac{(1+2 T) K_{2}}{T} \sum_{k=1}^{m}\left(T-t_{k}\right) \approx 0.9207589286<1 .
$$

Hence, by Theorem 3.4, boundary value problem (4.3) has at least one solution on $[0,1]$.

Example 4.4 Consider the following nonlinear second-order impulsive $q_{k}$-difference equation with separated boundary condition:

$$
\left\{\begin{array}{l}
D_{\frac{k+2}{k+4}}^{2} x(t)=\frac{x}{x^{2}+6 e^{t}+42}+\frac{1+\sin \pi t}{e^{t}+7}-\cos t \int_{t_{k}}^{t} \frac{(t-s) \sin t}{e^{t-s}+2} x(s) d_{q_{k}} s, \quad t \in J, t \neq t_{k}, \\
\Delta x\left(t_{k}\right)=\frac{\sin \pi x\left(t_{k}\right)}{8 \pi(k+7)}, \quad k=1,2, \ldots, 9, \\
D_{\frac{k+2}{k+4}} x\left(t_{k}^{+}\right)-D_{\frac{k+1}{k+3}} x\left(t_{k}\right)=\frac{x\left(t_{k}\right) \cos ^{2} k t}{8(k+11)}, \quad k=1,2, \ldots, 9, \\
x(0)+D_{\frac{1}{2}} x(0)=0, \quad x(1)+D_{\frac{11}{13}} x(1)=0 .
\end{array}\right.
$$

Here $J=[0,1], t_{k}=k / 10, q_{k}=(k+2) /(k+4)$ for $k=0,1,2, \ldots, 9, m=9, T=1, f(t, x)=$ $\left(x /\left(x^{2}+6 e^{t}+42\right)\right)+(1+\sin \pi t) /\left(e^{t}+7\right)-\cos t \int_{t_{k}}^{t}\left((t-s) \sin t /\left(e^{t-s}+2\right)\right) x(s) d_{q_{k}} s, I_{k}(x)=$ $(\sin \pi x) /(8 \pi(k+7))$, and $I_{k}^{*}(x)=\left(x \cos ^{2} k t\right) /(8(k+11))$. Clearly,

$$
\begin{aligned}
|f(t, x)| & =\left|\frac{x}{x^{2}+6 e^{t}+42}+\frac{1+\sin \pi t}{e^{t}+7}-\cos t \int_{t_{k}}^{t} \frac{(t-s) \sin t}{e^{t-s}+2} x(s) d_{q_{k}} s\right| \\
& \leq\left(\frac{2}{e^{t}+7}\right) \frac{|x+1|}{6}+\left|\int_{t_{k}}^{t} \frac{(t-s) \sin t}{e^{t-s}+2} x(s) d_{q_{k}} s\right| \\
\left|I_{k}(x)\right| & =\left|\frac{\sin \pi x}{8 \pi(k+7)}\right| \leq \frac{|x|}{64} \quad \text { and } \quad\left|I_{k}^{*}(x)\right|=\left|\frac{x \cos ^{2} k t}{8(k+11)}\right| \leq \frac{|x|}{96} .
\end{aligned}
$$

Choosing $p(t)=\frac{2}{e^{t}+7}, \psi(|x|)=\frac{|x+1|}{6}, \varphi_{1}(|u|)=\frac{|x|}{64}$, and $\varphi_{2}(|x|)=\frac{|x|}{96}$, we obtain

$$
\frac{M^{*}}{0.9854645864 M^{*}+0.1469016279}>1
$$

which implies that $M^{*}>10.10646356$. Hence, by Theorem 3.6, boundary value problem (4.4) has at least one solution on $[0,1]$.

\section{Competing interests}

The authors declare that they have no competing interests.

Authors' contributions

All authors contributed equally in this article. They read and approved the final manuscript.

\section{Authors' information}

Sotiris K Ntouyas is a member of Nonlinear Analysis and Applied Mathematics (NAAM)-Research Group at King Abdulaziz University, Jeddah, Saudi Arabia.

\section{Author details}

${ }^{1}$ Department of Mathematics, Faculty of Applied Science, King Mongkut's University of Technology, North Bangkok, Bangkok, Thailand. ${ }^{2}$ Department of Mathematics, University of loannina, loannina, 451 10, Greece. 


\section{Acknowledgements}

The research of C Thaiprayoon and J Tariboon is supported by King Mongkut's University of Technology North Bangkok, Thailand.

Received: 3 December 2013 Accepted: 28 February 2014 Published: 17 Mar 2014

\section{References}

1. Tariboon, J, Ntouyas, SK: Quantum calculus on finite intervals and applications to impulsive difference equations. Adv. Differ. Equ. 2013, 282 (2013)

2. Kac, V, Cheung, P: Quantum Calculus. Springer, New York (2002)

3. Bangerezako, G: Variational q-calculus. J. Math. Anal. Appl. 289, 650-665 (2004)

4. Dobrogowska, A, Odzijewicz, A: Second order q-difference equations solvable by factorization method. J. Comput. Appl. Math. 193, 319-346 (2006)

5. Gasper, G, Rahman, M: Some systems of multivariable orthogonal q-Racah polynomials. Ramanujan J. 13, 389-405 (2007)

6. Ismail, MEH, Simeonov, P: q-difference operators for orthogonal polynomials. J. Comput. Appl. Math. 233, 749-761 (2009)

7. Bohner, M, Guseinov, GS: The $h$-Laplace and q-Laplace transforms. J. Math. Anal. Appl. 365, 75-92 (2010)

8. El-Shahed, M, Hassan, HA: Positive solutions of q-difference equation. Proc. Am. Math. Soc. 138, 1733-1738 (2010)

9. Ahmad, B: Boundary-value problems for nonlinear third-order q-difference equations. Electron. J. Differ. Equ. 94, 1-7 (2011)

10. Ahmad, B, Alsaedi, A, Ntouyas, SK: A study of second-order q-difference equations with boundary conditions. Adv. Differ. Equ. 2012, 35 (2012)

11. Ahmad, B, Ntouyas, SK, Purnaras, IK: Existence results for nonlinear q-difference equations with nonlocal boundary conditions. Commun. Appl. Nonlinear Anal. 19, 59-72 (2012)

12. Ahmad, B, Nieto, JJ: On nonlocal boundary value problems of nonlinear q-difference equations. Adv. Differ. Equ. 2012, 81 (2012)

13. Ahmad, B, Ntouyas, SK: Boundary value problems for q-difference inclusions. Abstr. Appl. Anal. 2011, 292860 (2011)

14. Zhou, W, Liu, H: Existence solutions for boundary value problem of nonlinear fractional q-difference equations. Adv. Differ. Equ. 2013, 113 (2013)

15. Yu, C, Wang, J: Existence of solutions for nonlinear second-order $q$-difference equations with first-order q-derivatives. Adv. Differ. Equ. 2013, 124 (2013)

16. Lakshmikantham, V, Bainov, DD, Simeonov, PS: Theory of Impulsive Differential Equations. World Scientific, Singapore (1989)

17. Samoilenko, AM, Perestyuk, NA: Impulsive Differential Equations. World Scientific, Singapore (1995)

18. Benchohra, M, Henderson, J, Ntouyas, SK: Impulsive Differential Equations and Inclusions, vol. 2. Hindawi Publishing Corporation, New York (2006)

19. Krasnoselskii, MA: Two remarks on the method of successive approximations. Usp. Mat. Nauk 10, $123-127$ (1955)

20. Granas, A, Dugundji, J: Fixed Point Theory. Springer, New York (2003)

10.1186/1687-1847-2014-88

Cite this article as: Thaiprayoon et al.: Separated boundary value problems for second-order impulsive $q$-integro-difference equations. Advances in Difference Equations 2014, 2014:88

\section{Submit your manuscript to a SpringerOpen ${ }^{\ominus}$ journal and benefit from:}

$\rightarrow$ Convenient online submission

Rigorous peer review

- Immediate publication on acceptance

- Open access: articles freely available online

- High visibility within the field

- Retaining the copyright to your article 